Published in final edited form as:

Nature. 2017 August 31; 548(7669): 582-587. doi:10.1038/nature23663.

\title{
Lhx6-positive GABA-releasing neurons of the zona incerta promote sleep
}

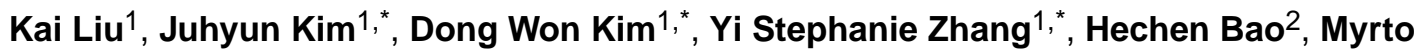 \\ Denaxa $^{3}$, Szu-Aun Lim ${ }^{2}$, Eileen Kim ${ }^{4}$, Chang Liu ${ }^{1}$, lan R. Wickersham ${ }^{5}$, Vassilis Pachnis ${ }^{3}$, \\ Samer Hattar ${ }^{6}$, Juan Song ${ }^{2}$, Solange P. Brown ${ }^{1}$, and Seth Blackshaw ${ }^{1}$ \\ ${ }^{1}$ Solomon H. Snyder Department of Neuroscience, Johns Hopkins University School of Medicine, \\ Baltimore, Maryland, USA \\ 2Department of Pharmacology, University of North Carolina School of Medicine, Chapel Hill, \\ North Carolina, USA \\ ${ }^{3}$ The Francis Crick Institute, London, UK \\ ${ }^{4}$ Department of Biology, Johns Hopkins University, Baltimore, Maryland, USA \\ ${ }^{5}$ The McGovern Institute for Brain Research, Massachusetts Institute of Technology, Cambridge, \\ Massachusetts, USA \\ ${ }^{6}$ National Institute of Mental Health, Bethesda, Maryland, USA
}

\section{Abstract}

Multiple populations of wake-promoting neurons have been characterized in mammals, but few sleep-promoting neurons have been identified ${ }^{1}$. Wake-promoting cell types include hypocretin and GABA ( $\gamma$-aminobutyric-acid)-releasing neurons of the lateral hypothalamus, which promote the transition to wakefulness from non-rapid eye movement (NREM) and rapid eye movement (REM) sleep $^{2,3}$. Here we show that a subset of GABAergic neurons in the mouse ventral zona incerta, which express the LIM homeodomain factor Lhx6 and are activated by sleep pressure, both directly inhibit wake-active hypocretin and GABAergic cells in the lateral hypothalamus and receive inputs from multiple sleep-wake-regulating neurons. Conditional deletion of Lhx6 from the developing diencephalon leads to decreases in both NREM and REM sleep. Furthermore, selective activation and inhibition of Lhx6-positive neurons in the ventral zona incerta bidirectionally regulate sleep time in adult mice, in part through hypocretin-dependent

\footnotetext{
Reprints and permissions information is available at www.nature.com/reprints.

Correspondence and requests for materials should be addressed to S.B. (sblack@jhmi.edu).

These authors contributed equally to this work.

Supplementary Information is available in the online version of the paper.

Author Contributions

K.L. and S.B. designed the study. K.L., D.W.K. and Y.S.Z. conducted immunostaining. C.L. conducted statistical analysis. J.K. and S.P.B. designed, conducted and analysed slice electrophysiology experiments. H.B., S.-A.L and J.S. performed rabies virus experiments. I.R.W. provided rabies virus. M.D. and V.P. generated $L h x \sigma^{\text {lox } / l o x}$ mice. K.L. performed all other surgeries and behavioural analysis, with assistance from D.W.K. and E.K., under the guidance of S.H. K.L., D.W.K., J.K., S.H., S.P.B. and S.B. wrote the paper.

The authors declare no competing financial interests.
} 
mechanisms. These studies identify a GABAergic subpopulation of neurons in the ventral zona incerta that promote sleep.

Sleep-wake transitions are rapid, bistable and controlled by a distributed network of sleepand wake-promoting neurons ${ }^{3}$. The lateral hypothalamus contains several subtypes of wakepromoting neurons, including glutamatergic hypocretin (Hcrt)-expressing neurons and GABAergic neurons ${ }^{2,3}$. Multiple inhibitory neuronal subtypes innervate $\mathrm{Hcrt}^{+}$lateral hypothalamus neurons ${ }^{4}$, but their roles in regulating sleep remain unclear. The zona incerta, which is adjacent to the lateral hypothalamus, regulates sensory-motor integration and sends efferent projections to multiple brain regions ${ }^{5}$. Stimulation of thalamic afferents to the zona incerta can induce a sleep-like state ${ }^{6}$. Although bilateral ablation of the zona incerta does not affect sleep ${ }^{7}$, the role of individual zona incerta cell types in controlling sleep is unknown.

We previously observed Lhx6 expression in a subset of GABAergic neuronal precursors in the developing mouse hypothalamus from embryonic day (E) 11.5 onwards $^{8}$. We identified an eGFP transgenic line ${ }^{9}$ that recapitulated native expression of Lhx6 in the brain (98\% of $\mathrm{eGFP}^{+}$cells are Lhx6 ${ }^{+}$; Extended Data Fig. 1a-e). In diencephalon, Lhx6 was expressed in a zone extending from the ventral zona incerta (VZI), through the dorsomedial hypothalamic nucleus and lateral hypothalamus, to the posterior hypothalamus ${ }^{8}$ (Fig. 1a, b and Extended Data Fig. 1a-d). To study these cells, we used Lhx6-cre transgenic mice ${ }^{10}$, which showed co-expression of Lhx6 protein and tdTomato when crossed with the $A i 9$ reporter (Extended Data Fig. 2a-e).

Hypothalamic Lhx6 $6^{+}$cells express markers of GABAergic neurons, including Gad1 and Slc32a1 (Fig. 1e-g and Extended Data Fig. 1f-i). Although Lhx6 is expressed in parvalbumin-positive $\left(\mathrm{Pvalb}^{+}\right)$and somatostatin-positive $\left(\mathrm{Sst}^{+}\right)$cortical interneurons, and is necessary for their differentiation ${ }^{11}$, we observed no colocalization of Lhx6 with either Pvalb or Sst in the zona incerta or lateral hypothalamus (Extended Data Fig. 1j-q). Additionally, Lhx6 $6^{+}$cells in the lateral hypothalamus expressed neither Hcrt nor the sleeppromoting neuropeptide melanin-concentrating hormone $(\mathrm{MCH})^{1}$ (Extended Data Fig. 2f$\mathrm{k})$.

Lhx $6^{+}$cells represent $45 \%$ and $33 \%$ of all Slc32a $1^{+}$and Gad $1^{+}$cells in the VZI (Fig. 1c, d). Following AAV9-DIO-ChR2-eYFP virus injections into the zona incerta of Lhx6-creER ${ }^{T 2}$ knock-in mice ${ }^{12}$ (Fig. 1h), we observed local projections within the zona incerta and to Hcrt ${ }^{+}$neurons in the lateral hypothalamus (Fig. 1i, j). Projections to monoaminergic midbrain populations were seen, including to the substantia nigra, the ventral tegmental area and the dorsal raphe nucleus, as well as to the ventrolateral periaqueductal grey and the central amygdala (Extended Data Fig. 3b, e, h, k, n). These overlap with known projections of GABAergic neurons of the zona incerta ${ }^{13}$ (Extended Data Fig. 3c, f, i, 1, o), but differ in that no projections from Lhx6 neurons of the zona incerta to the thalamus - the main target of Pvalb $^{+}$GABAergic neurons of the zona incerta-were seen (Extended Data Fig. 3d, g, j, m, p).

To map inputs to Lhx6 $6^{+}$VZI neurons, we conducted retrograde monosynaptic tracing using rabies virus ${ }^{14}$, and observed labelling in many brain regions, including reciprocal 
connections between all regions innervated by Lhx $6^{+}$VZI neurons, along with other regions that control sleep. $\mathrm{Lhx}^{+}$VZI neurons appear to receive inputs from wake-active and REMinhibiting neurons, including dopaminergic neurons of the ventral tegmental area, serotoninergic neurons of the dorsal raphe nucleus, and GABAergic neurons of both the ventrolateral periaqueductal grey and lateral hypothalamus ${ }^{1,3,15}$ (Fig. 1k, o-t and Extended Data Fig. 4). Lhx6 ${ }^{+}$VZI neurons also receive projections from REM-promoting cholinergic neurons of the basal forebrain and pedunculopontine nucleus ${ }^{1,3}$ (Fig. 11-n and Extended Data Fig. 4). Finally, we observed inputs from other regions that have not previously been implicated in sleep regulation (Extended Data Fig. 4).

We next tested whether Lhx6 $6^{+}$VZI neurons were activated during sleep. We observed higher Fos expression at lights on, when sleep pressure is highest, relative to lights off, when sleep pressure is lowest ${ }^{16}$ (Fig. 2a-e). We then showed that 6-h sleep deprivation from zeitgeber time (ZT)0 (lights on) to ZT6-either alone or in combination with $1 \mathrm{~h}$ of recovery sleep from ZT6 to ZT7-increased Fos expression in Lhx6 ${ }^{+}$VZI neurons (Fig. 2f-h), demonstrating that Lhx6 $6^{+}$VZI neurons are activated by high sleep pressure.

To test whether Lhx6 ${ }^{+}$VZI neurons directly inhibit wake-promoting $\mathrm{Hcrt}^{+}$neurons in the lateral hypothalamus, we performed whole-cell patch-clamp recordings in brain slices from Lhx6-cre;Hcrt-eGFP mice ${ }^{17}$ infected with AAV9-DIO-ChR2-mCherry (Extended Data Fig. $5 a)$. Cherry $^{+}$neurons fired action potentials in current-clamp mode following photostimulation (Fig. 2i). Photostimulation of Lhx6 ${ }^{+}$VZI neurons induced short-latency postsynaptic responses $(1.82 \pm 0.22 \mathrm{~ms} ; n=21)$ in $\mathrm{Hcrt}-\mathrm{eGFP}^{+}$neurons recorded using high-chloride internal solution (Fig. 2j, k and Extended Data Fig. 5b, e-g). These responses were unaffected by ionotropic glutamate receptor antagonists, and eliminated by the $\mathrm{GABA}_{\mathrm{A}}$ receptor antagonist gabazine (Fig. 2k, 1). Responses recorded with co-application of the potassium channel blocker 4 -AP and tetrodotoxin ${ }^{18}$ indicate that Lhx $6^{+}$VZI neurons form monosynaptic connections onto Hcrt-eGFP ${ }^{+}$neurons (Extended Data Fig. 5c, d).

We also tested whether Lhx6 ${ }^{+}$VZI neurons inhibited wake-promoting GABAergic neurons in the lateral hypothalamus ${ }^{15}$, using $L h x 6$-cre;Gad2-NLS-mCherry mice and the approach described above. The Gad2-mCherry ${ }^{+}$cells showed a range of firing patterns following current steps, as described ${ }^{19}$ (Fig. $2 \mathrm{~m}-$ p). Gad2-mCherry ${ }^{+}$neurons in the lateral hypothalamus exhibited short-latency postsynaptic responses following photostimulation of Lhx6 $6^{+}$VZI neurons, which were eliminated by gabazine, similar to $\mathrm{Hcrt}^{+}$neurons $(1.83$ $\pm 0.19 \mathrm{~ms}, n=10$; Fig. $2 \mathrm{~m}-\mathrm{p}$ and Extended Data Fig. $5 \mathrm{~h}-1$ ).

Reciprocal connections among GABAergic neurons can facilitate transitions between bistable activity states, including sleep-wake transitions ${ }^{3,20,21}$. We tested whether Lhx $6^{+}$ VZI GABAergic neurons were interconnected, using Lhx6-creER ${ }^{T 2}$;Lhx6-eGFP mice and the approaches described above (Extended Data Fig. 5m-u). We observed short-latency postsynaptic potentials in a subset of $\mathrm{Lhx}^{+}$cells, indicating interconnectivity (Extended Data Fig. 5q, r).

We next selectively deleted Lhx6 in diencephalon ${ }^{22}$ (Fig. 3a). Foxd1-cre; Lhx $6^{\text {lox/lox }}$ mice show loss of Lhx6 immunostaining in the hypothalamus, but not in the telencephalon 
(Extended Data Fig. 6a-1). Foxd1-cre;Lhx $6^{\text {lox/lox }}$ mice show no gross behavioural abnormalities (Extended Data Fig. 6q-v). However, electroencephalogram (EEG) and electromyography (EMG) data from Foxd1-cre;Lhx $6^{l o x / l o x}$ mice revealed an increase in wake time, and a reduction in NREM and REM sleep, relative to controls (Fig. 3b). Spectral analysis of EEGs from Foxd1-cre;Lhx $\sigma^{\text {lox/lox }}$ mice showed a reduction in delta waves during NREM sleep (Fig. 3c, d) and an increase in alpha waves during wake (Fig. 3e, f). The relative decrease of REM sleep was greater than that of NREM sleep. Further analysis revealed an increase in wake bout length in Foxd1-cre; Lhx $\sigma^{\text {lox } / l o x}$ mice (Extended Data Fig. 6o, p). This shows that selective deletion of Lhx6 from the diencephalon resulted in decreased sleep.

Using DREADD (designer receptors exclusively activated by designer drugs)-based chemogenetic analysis ${ }^{23}$, we next tested whether activation and inhibition of Lhx6 ${ }^{+}$VZI neurons modulated sleep. In Lhx6-cre mice infected bilaterally with $\mathrm{G}_{\mathrm{q}}$-coupled DREADDs, clozapine $\mathrm{N}$-oxide (CNO) induced Fos expression in zona incerta cells (Fig. $4 \mathrm{a}, \mathrm{b}$ and Extended Data Fig. 7h-k), and did not affect sleep in mice expressing mCherry in Lhx6 ${ }^{+}$ VZI neurons (Extended Data Fig. 7p-r). We next tested whether activation of Lhx6 $6^{+}$VZI neurons induced sleep. We observed an increase in REM sleep during the $12 \mathrm{~h}$ following CNO injection at all times except ZT23, when sleep pressure is highest. Decreases in wake, and increases in NREM sleep, were observed following CNO administration at ZT5 and ZT11 (Fig. 4c, d and Extended Data Fig. 8). These effects were strongest between 2 and $8 \mathrm{~h}$ following CNO administration, and were gone after $12 \mathrm{~h}$. CNO injection at ZT5 increased delta waves during NREM sleep and decreased theta and gamma waves during wake (Extended Data Fig. 8). Selective transduction of Lhx6 $6^{+}$VZI neurons was confirmed by postmortem examination (Extended Data Fig. 7a-g).

To determine whether activation of Lhx $6^{+}$VZI neurons promotes sleep by inhibiting $\mathrm{Hcrt}^{+}$ neurons, we performed a pharmacological occlusion experiment, where we activated Lhx $6^{+}$ zona incerta neurons while applying the dual hypocretin receptor antagonist suvorexant ${ }^{24}$. As reported, suvorexant rapidly increased both NREM and REM sleep, but these effects were lost by $4 \mathrm{~h}$ (Extended Data Fig. 9). Combining CNO and suvorexant resulted in levels of wake and of NREM sleep, but not of REM sleep, indistinguishable from those seen with suvorexant alone, indicating that $\mathrm{Lhx}^{+}$zona incerta neurons control Hcrt release to promote NREM, but not REM, sleep.

Finally, we show that inhibition of Lhx6 ${ }^{+}$VZI neurons with $\mathrm{G}_{\mathrm{i}}$-coupled DREADDs reduced sleep (Fig. 4a, e-g and Extended Data Fig. 10). CNO administration at ZT5 and ZT11 reduced time spent in REM sleep. A decrease in time spent in NREM sleep, and corresponding increase in time awake, were also observed following CNO administration at ZT5 (Fig. 4f), along with a decrease in delta waves during NREM sleep (Extended Data Fig. 10). CNO administered at ZT5 inhibited NREM and REM sleep, and increased wake, for up to $8 \mathrm{~h}$ following injection (Fig. $4 \mathrm{~g}$ ). No significant change was seen in total time spent in wake, NREM or REM sleep in the $12 \mathrm{~h}$ following CNO injection in the middle or the end of the dark phase at ZT17 and ZT23 (Extended Data Fig. 10). When data were examined in two-hour bins, decreases in NREM and REM sleep, and a corresponding increase in wake, were observed $8-10 \mathrm{~h}$ following $\mathrm{CNO}$ administration. These decreases occurred during the 
beginning of the light phase (Extended Data Fig. 10), suggesting that the sleep-inhibiting effects of Lhx6 $6^{+}$VZI neurons are confined to the normal sleep time of mice (light phase) (Fig. 2h).

In this study, we identify GABAergic Lhx6 ${ }^{+}$VZI neurons as a subpopulation of sleeppromoting neurons. These join a handful of other GABAergic sleep-promoting neuronal populations, including cells in the ventrolateral preoptic area and the parafacial zone of the medulla ${ }^{25-27}$. Lhx6 $6^{+}$VZI neurons differ from these sleep-promoting cells in that they promote both NREM and REM sleep, rather than either alone. They also exhibit a relatively slow-onset but long-lasting regulation of sleep, which may be related to their interconnectivity, a property that they share with other GABAergic zona incerta neurons ${ }^{28}$. Lhx $6^{+}$VZI neurons receive inputs from multiple different subtypes of sleep-regulating neurons, and are directly presynaptic to wake-promoting GABAergic and $\mathrm{Hcrt}^{+}$neurons of the lateral hypothalamus and inhibit their activity, with this latter function being essential for their promotion of NREM sleep. Since complete ablation of the zona incerta does not induce changes in sleep-wake cycles ${ }^{7}$, and global activation of GABAergic neurons in the zona incerta does not affect sleep ${ }^{15}$, these findings imply that other wake-promoting GABAergic subpopulations exist in the zona incerta.

\section{Methods}

\section{Mice}

All experimental animal procedures were approved by the Johns Hopkins University Institutional Animal Care and Use Committee. The BAC transgenic Lhx6-eGFP mouse line was generated as part of the GENSAT project ${ }^{9}$ and obtained from the MMRRC (stock number: 000246). Hcrt-eGFP transgenic mice were obtained from L. de Lecea (Stanford University School of Medicine) ${ }^{17}$. Lhx6-cre mice were shared by A. Gittis (Carnegie Mellon University) and were originally generated by N. Kessaris (University College London) ${ }^{10}$. Lhx6-creER ${ }^{T 2}$ knock-in mice were generated by Z. J. Huang (Cold Spring Harbour Laboratories) ${ }^{12}$ and were purchased from The Jackson Laboratory (stock number: 010776). Foxd1-cre-eGFP knock-in mice ${ }^{29}$ (stock number: 012463) and Gad2-T2a-NLS-mCherry mice $^{30}$ (stock number: 023140) were obtained from The Jackson Laboratory.

The conditional $L h \times 6$ allele was generated through homologous recombination using a targeting construct in which loxP sites were placed in non-coding regions that are $5^{\prime}$ to coding exon $1 \mathrm{~b}$ and $3^{\prime}$ to coding exon 3 . The $5^{\prime}$ homology consists of a 3.1-kb XbaI-SacI fragment containing the $5^{\prime}$ upstream region and the first exon (1a) of $L h x 6$, whereas the $3^{\prime}$ homology consists of a 5.4-kb ApaI-NheI fragment. For the Lhx 6 -targeting vector, the $2-\mathrm{kb}$ genomic fragment between the homology regions is replaced by a 4-kb cassette, containing the following: (1) the 1b, 2 and 3 coding exons flanked by lox $P$ sites; (2) the neomycinresistance gene under the control of the phosphoglycerate kinase (PGK) promoter (PGKNeo) flanked by FRT sites. Targeting constructs were linearized and electroporated into E14Tg2A embryonic stem cells. Targeted clones were identified and analysed in detail by Southern blotting using $5^{\prime}$ and $3^{\prime}$ external probes. Germline transmission of the mutant alleles was achieved using standard protocols. The phenotypic analysis presented was performed on mice from which the PGK-Neo cassette was removed by crossing founder 
Lhx $\sigma^{\text {lox/lox }}$ mice with the $A c t b:: F I P e$ transgenic line ${ }^{31}$. For the maintenance of the Lhx $\sigma^{\text {lox/lox }}$ colony, we performed PCR using the following primers: Lhx6flR:

GGAGGCCCAAAGTTAGAACC Lhx6flF: CTCGAGTGCTCCGTGTGTC.

\section{Behavioural analysis of Foxd1-cre;Lhx6/ox/lox mice}

We conducted several behavioural tests to investigate the phenotype of Foxd1-

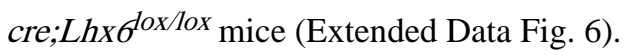

Light-dark preference test-Each mouse was placed in an apparatus consisting of a dark chamber and a light-illuminated chamber. Mice could move freely between the two chambers. The duration each mouse spent in the dark chamber, light chamber or centre was measured and normalized relative to the entire experimental period.

Elevated plus maze (EPM)—Each mouse was placed in the centre of the EPM and videotaped for $5 \mathrm{~min}$. The time spent in the open and closed arms was determined from the video recording by blinded observers.

Open field test-Mice were placed in the open field chamber and time spent in the centre and periphery was assessed for $30 \mathrm{~min}$, using a Photobeam activity system to measure beam breaks.

Rotarod-Up to four mice were tested at one time on a rotating rod with the capacity to gradually accelerate up to 99.9 revolutions per minute (r.p.m.). A photobeam activity system was used to record when the mouse dropped off of the rod as the rotation speed gradually increased. The average terminal rotation speed and time spent on the Rotarod was used for analysis.

Grip strength-Forelimb grip strength was measured as tension force using a computerized grip strength meter (GSM). Mice were lifted over the baseplate by their tails, and their forepaws allowed to grasp the steel grip. The tail of each mouse was then placed in the centre of the Single Axis Grip Strength Alignment Tool (SAGSAT) and the mouse was then gently pulled backward by the tail until its grip was released. The GSM was then used to measure the maximal force before the mouse released the bar. Three trials were performed for each mouse with a 1-min resting period between trials.

\section{Fos analysis}

To characterize zona incerta Lhx6 neurons activated under different lighting conditions, adult Lhx6-eGFP mice (2-4 months old) were deeply anaesthetized with ketamine at four time points across the light-dark cycle: 07:00 (ZT0); 13:00 (ZT6); 21:00 (ZT14, lights off); 01:00 (ZT18). For the 07:00 time point, mice were anaesthetized a few minutes before lights on with minimal light exposure. All other mice were anaesthetized within $15 \mathrm{~min}$ of their time-point group. Immediately after anaesthesia, mice were transcardially perfused with 20 $\mathrm{ml} 1 \times$ phosphate-buffered saline (PBS) followed by $4 \%$ paraformaldehyde (PFA) $(\mathrm{m} / \mathrm{v})$ in $1 \times$ PBS. For analysis of Fos expression during sleep deprivation, adult Lhx6-eGFP mice (24 months old) were sleep-deprived starting at 07:00 (ZT0) until 13:00 for a total of $6 \mathrm{~h}$. To 
facilitate sleep deprivation, EEG-implanted mice were monitored in new cages with new bedding, given water and food ad libitum, and occasionally touched gently with a soft brush when they seemed drowsy, as previously described ${ }^{32}$. Control mice were not disturbed and allowed to sleep freely. Mice in the sleep deprivation group and control group were anaesthetized at 13:00 and transcardially perfused and stained according to the protocol for immunohistochemistry. Mice in the sleep deprivation with recovery sleep group were permitted to sleep at 13:00 for $1 \mathrm{~h}$ and then perfused at 14:00.

\section{Cell counting}

For quantification of co-localization of Lhx6 and other histological markers, the area used for counting was demarcated by the Lhx6 cells in the zona incerta that were labelled by Lhx6-eGFP. All cell counting was conducted blind on $3 \times 2$ tiled images of the zona incerta using ImageJ. For each mouse, brain sections were analysed bilaterally, and the percentage of marker-positive neurons in Lhx6-eGFP ${ }^{+}$neurons was compared by nonparametric statistical methods (see 'Statistics').

\section{Stereotaxic AAV injection and EEG/EMG implantation}

Surgery-AAV vectors were obtained from vector cores of the University of Pennsylvania (AAV9-EF1a. DIO.hChR2-eYFP) ${ }^{33}$ (Fig. 1 and Extended Data Fig. 4) and University of North Carolina (AAV9-EF1a.DIO.hM3Dq-mCherry; AAV9- EF1a.DIO.hM3Dq-eYFP; AAV9-EF1a.DIO.hM4Di-mCherry) ${ }^{23}$. For bilateral stereotaxic injection of AAV into the zona incerta, adult male mice (2-4 months old) were anaesthetized with ketamine/xylazine (100 $\mu \mathrm{l}$ of $100 \mathrm{mg} \mathrm{ml}^{-1}$ ketamine, $20 \mathrm{mg} \mathrm{ml}^{-1}$ xylazine) and placed in a stereotaxic frame. The scalp was incised to expose the skull and connective tissue was gently scraped away. After exposing the skull, bilateral craniotomies ( $\sim \mathrm{mm}$ diameter each) were made to allow

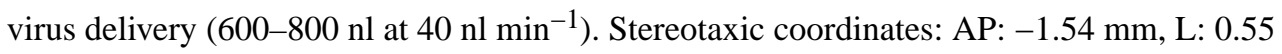
$\mathrm{mm}, \mathrm{DV}:-4.8 \mathrm{~mm}$, according to the reference atlas in ref. 34 . Skin covering the boreholes was sutured closed following surgery. Lhx6-creER ${ }^{T 2}$ mice that were injected with ChR2eYFP constructs to map postsynaptic projections of Lhx6 VZI neurons, beginning one week following viral injection, $1.0 \mathrm{mg}$ of 4-hydroxytamoxifen dissolved in corn oil was administered via intraperitoneal injection once a day for a total of five days in order to activate expression of the Cre-dependent channel-rhodopsin (ChR2) construct.

For EEG/EMG recordings, four-channel tethered EEG/EMG biosensors (Pinnacle Technology Inc.) were anchored onto the mouse skull. The two front screws used for EEG1 signal recording were positioned at: AP: $+2 \mathrm{~mm}, \mathrm{ML}: \pm 1.5 \mathrm{~mm}$. The two back screws used for the EEG2 signal recording were positioned at: bregma AP: $-4 \mathrm{~mm}, \mathrm{ML}: \pm 1.5 \mathrm{~mm}$. With the same implant, two EMG antenna electrodes were inserted into the left and right neck muscles. The EEG/EMG recording implant was secured to the mouse skull with dental cement. For EEG/EMG analysis of DREADD-injected mice, this surgery was performed immediately following AAV injection.

EEG/EMG recording-For all analysis of sleep behaviour, the Pinnacle Technology EEG/EMG tethered recording system for sleep behaviour evaluation was used, and mice were allowed a minimum of 10 days recovery in a 12-h light-dark cycle following 
EEG/EMG biosensor implantation. Implanted mice were tethered to a $100 \times$ preamplifier (Pinnacle Technology) and were housed in a 12-h dark-12-h light cycle (lights on between 07:00 (ZT0) and 19:00 (ZT12)) recording chamber. The mice were allowed around 5-7 days to acclimatize to the recording chamber before recording. The EEG and EMG signals were preamplified 5,000x and digitized at 14 bits and then recorded. Signal acquisition was obtained using the Sirena acquisition suite (Pinnacle Technology Inc.). EEG/EMG traces were recorded for $48 \mathrm{~h}$ for Foxd1-cre; $L h x \sigma^{\text {lox } / l o x}$ mice, and data from the second day extracted for analysis (Fig. 3 and Extended Data Fig. 6). For analysis of mice injected with AAV-expressing cre-dependent $\mathrm{G}_{\mathrm{q}^{-}}$and $\mathrm{G}_{\mathrm{i}}$-linked DREADD constructs, we used the virustransfected mice with saline injection as control compared to mice with clozapine- $N$-oxide (CNO, Sigma-Aldrich; $0.5 \mathrm{mg} \mathrm{kg}^{-1}$ in saline) intraperitoneal injection. The following injection protocol was used for analysis of all mice injected with AAV-expressing Credependent DREADD constructs: on day 1, we performed two consecutive saline injections at 12-h intervals at ZT23 (06:00) and ZT11 (18:00); on day 2, we injected CNO at ZT23 and ZT11. Alternatively, on day 1, the saline was injected at ZT5 and ZT17, and CNO at the same times on day 2 (Fig. 4 and Extended Data Figs 8-10). For analysis of mice injected with AAV-expressing Cre-dependent $\mathrm{G}_{\mathrm{q}}$ DREADDs with suvorexant, a dual orexin receptor antagonist ${ }^{35}$, we used the virus-transfected mice with saline injection as controls. These were directly compared to mice that were administered $\mathrm{CNO}$ by intraperitoneal injection; suvorexant (Selleckchem; $25 \mathrm{mg} \mathrm{kg}^{-1}$ in $20 \%$ TPGS) was administered by oral gavage as described previously ${ }^{24}$; or mice were simultaneously treated with $\mathrm{CNO}$ and suvorexant. The following injection protocol was used for analysis of all mice injected with AAV-expressing Cre-dependent DREADDs: on day 1, we performed one saline injection at ZT5 (12:00); on day 2, we injected CNO at ZT5; on day 3, we injected suvorexant at ZT5; on day 5, we injected CNO and suvorexant at ZT5 (Extended Data Fig. 9).

To validate the specificity and efficacy of AAV DREADD infection following behavioural analysis, mice were given a single injection of $\mathrm{CNO}\left(0.5 \mathrm{mg} \mathrm{kg}^{-1}\right.$ in saline $)$ and euthanized $1 \mathrm{~h}$ later by terminal anaesthesia and transcardiac perfusion. For $\mathrm{G}_{\mathrm{q}}$ DREADDs mice, $\mathrm{CNO}$ was injected at ZT5, and for $\mathrm{G}_{\mathrm{i}}$ DREADDs mice, CNO was injected between ZT8 and ZT9. Immunohistochemistry was then performed to detect both mCherry and Fos expression (Extended Data Fig. 7).

EEG/EMG analysis—Raw EEG/EMG signals were loaded into Neuroscore (DSI) for scoring. We set the high-pass filter at $0.5 \mathrm{~Hz}$ and the low-pass filter at $40 \mathrm{~Hz}$ for both EEG channels. EMG signals were high-pass filtered at $10 \mathrm{~Hz}$ and low-pass filtered at $100 \mathrm{~Hz}$. EEG1 and EEG2 signals were further transformed to be temporal AR (autoregressive model) spectrums. Researchers were blinded to genotype and/or treatment before data analysis. Sleep-wake state was visually scored in $~ 10$-s periods as either wake (low-voltage, highfrequency EEG wave with high activity of EMG), rapid eye movement (REM, low-voltage, prominent theta frequency in EEG channel and low EMG) or NREM (high-voltage, lowfrequency EEG wave with low activity of EMG). The percentage of time spent in each behavioural state was then statistically evaluated.

For investigating the sleep-wake behaviour of Foxd1-cre;Lhx $\sigma^{\text {lox/lox }}$ mice, the EEG/EMG analysis was conducted for $24 \mathrm{~h}$ for both Foxd1-cre;Lhx $6^{\text {lox/+ }}$ control and Foxd1- 
cre;Lhx $6^{\text {lox/lox }}$ mice (Fig. 3 and Extended Data Fig. 6). In our analysis of sleep-wake behaviour in mice expressing activating or silencing DREADDs, we analysed data for $12 \mathrm{~h}$ after either saline or CNO injection (Fig. 4), as effects of CNO were not observed at time points after $10 \mathrm{~h}$ post-injection. A 2-h accumulation analysis was also conducted for evaluating temporal dynamics of sleep-wake states.

Sleep deprivation was performed on EEG/EMG chip-implanted mice as previously described ${ }^{9}$ and EEG/EMG signals were recorded simultaneously for $6 \mathrm{~h}$ of sleep deprivation and $1 \mathrm{~h}$ of recovery sleep. The fraction of time spent asleep or awake in each stage during this period was quantified as described above to confirm that the mice were kept awake during the $6 \mathrm{~h}$ of sleep deprivation and went to sleep during the $1 \mathrm{~h}$ sleep recovery. For the $\mathrm{G}_{\mathrm{q}}$ DREADDs plus suvorexant drug experiment, we conducted EEG/EMG recordings and analysis as described above to quantify the duration of each sleep-wake stage when treated with $\mathrm{CNO}$, suvorexant or both drugs simultaneously.

For spectral band analysis, we used a previously described methodology ${ }^{15}$. We applied FFT to 5-s epochs of raw EEG waves from extracted NREM or wakefulness episodes separately to calculate EEG power frequency within the frequency range of $0.1-125 \mathrm{~Hz}$. On the basis of the sleep-wake stage labelling, we extracted a 2-h period of raw EEG wave of NREM or wake episodes between ZT7-ZT9 for both Lhx6 cKO and control mice for EEG power spectrum analysis. Similarly, in DREADD experiments, we extracted raw EEG waves of NREM or wake episodes between 2 and $4 \mathrm{~h}$ (ZT7-ZT9) after injection with saline or CNO at ZT5 for EEG power spectrum analysis to evaluate the effect of activating or silencing Lhx6 zona incerta neurons. Artefacts were removed from analysis by visual inspection of raw EEG and EMG data. In particular, data epochs that contained noise associated with large movements (EMG muscle artefacts) that occurred during active wake and vigilance states, which cover $\sim 10 \%$ of the recording interval, were manually flagged and excluded from analysis. Movements were confirmed by analysis of the videotape. In addition, a low-rank approximation was applied to remove the 5\% smallest Eigen factors from the raw data, and similar results were obtained to those seen using manual removal of artefacts.

The frequency data was collapsed into $0.5 \mathrm{~Hz}$ bins. All spectrum data were averaged for each bin for NREM or wake episodes separately. We then standardized the spectrum data by expressing each frequency bin from NREM or wake episodes for Lhx6 cKO or control mice as a percentage, relative to the same frequency bin and sleep stage in a baseline condition for the same mouse and at the same time of a different day (same zeitgeber time) (Fig. 3c-f). For DREADD experiments, we standardized the spectrum data by expressing each frequency bin in either saline or the $\mathrm{CNO}$ condition as a percentage, relative to the same frequency bin and sleep stage for a baseline condition for the same mouse and at the same time on a different day (same zeitgeber time) (Extended Data Figs 8, 10). The standardized frequency data were then averaged among each group of mice. To analyse the EEG frequency bands, relative power bins were summed as follows: delta $0.5-4 \mathrm{~Hz}$, low theta 4-6 $\mathrm{Hz}$, high theta $6-10 \mathrm{~Hz}$, alpha $10-20 \mathrm{~Hz}$, beta $20-40 \mathrm{~Hz}$ and gamma $40-100 \mathrm{~Hz}$. All the frequency bins and bands analyses were done using custom software written using MATLAB (MathWorks). 
We applied bout length and state transition analysis for all EEG/EMG physiology data (Extended Data Figs 6, 8,10). The sleep-wake stages bout lengths and transition counts were extracted from the same 2-h recording period (ZT7-ZT9) by reading the sleep bout reports (Neuroscore, DSI) automatically with a coding script written using MATLAB (Mathworks). The sleep-wake stage bout lengths and state transitions were averaged for each group of mice.

\section{Monosynaptic retrograde tracing with rabies virus}

Virus production-The rabies viral vector RV $\Delta \mathrm{G}-4 \mathrm{mCherry}($ EnvA) was made as described $^{36}$. In brief, HEK 293T cells (ATCC CRL-11268) were transfected with expression vectors for the ribozyme-flanked viral genome ( $\mathrm{pRV} \Delta \mathrm{G}-4 \mathrm{mCherry}$ ), rabies viral genes (pTIT-N, pTIT-P, pTIT-G and pTIT-L), and the T7 polymerase (pCAG-T7Pol). Supernatants were collected from 4 to 7 days after transfection, filtered, and pooled, passaged 3-4 times in BHK-B19G2 cells ${ }^{37}$ at a multiplicity of infection of 2-5, then passaged in BHK-EnvA2 cells ${ }^{37}$ at a multiplicity of infection of 2 . Purification, concentration and titration were done as described ${ }^{36,37}$.

Adeno-associated viral vector genome plasmid pAAV-Syn-FLEX-splitTVAeGFP-tTA was made from pAAV-Syn-FLEX-sTpEpB ${ }^{38}$ by cloning the tetracycline transactivator gene in place of the SAD B19 glycoprotein gene and eliminating the BamHI and NcoI sites in the P2A sequences by seamless cloning. Adeno-associated viral vector genome plasmid pAAVTREtight-BFP2-B19G was made by cloning the 'TRE-tight' tet response element from pAAV-TRE-HTG ${ }^{39}$, the mTagBFP2 gene ${ }^{40}$, a P2A element and the SAD B19 glycoprotein gene into pAAV-Syn-FLEX-sTpEpB ${ }^{38}$ by seamless cloning. Genomes were packaged into serotype 1 AAV by the University of North Carolina Vector Core. These AAV genome plasmids and their complete sequences have been deposited with Addgene with accession numbers 100798 and 100799.

Surgery-Monosynaptic retrograde tracing using rabies virus was performed as described $^{14,38}$. Adult Lhx6-cre mice were anaesthetized under 1-2\% isoflurane in oxygen with a $0.6-0.81$ per minute flow rate in a stereotaxic apparatus (Kopf). Virus was injected with a microsyringe (Hamilton, $33 \mathrm{GA}$ ) and microinjection pump (Hamilton) (rate at $100 \mathrm{nl}$ $\mathrm{min}^{-1}$ ). For rabies virus-based retrograde tracing, mice were injected with $500 \mathrm{nl}$ 1:1 mixed AAV1-Syn-FLEX-splitTVA-eGFP-tTA and AAV1-TREtight-BFP2-B19G in the right zona incerta (AP: $-1.5 \mathrm{~mm}$, ML: $+0.5 \mathrm{~mm}$, DV: $-4.75 \mathrm{~mm}$ ). After one week, the same mice received the second injection of $300 \mathrm{nl}$ pseudotyped rabies virus $\mathrm{RV} \Delta \mathrm{G}-4 \mathrm{mCherry}($ EnvA) using the same coordinates. Control mice were injected with RV $\Delta \mathrm{G}-4 \mathrm{mCherry}(\mathrm{EnvA})$ alone, with helper virus omitted. Then mice were transferred to a quarantined cubicle for special housing and monitoring. Seven days after rabies injection, mice were anaesthetized with ketamine and perfused with ice-cold 4\% PFA in PBS. Brains were collected and placed in $4 \%$ PFA overnight and switched to $30 \%$ sucrose for 2-3 days until they were fully sunk. Brains were sectioned on a microtome at a thickness of $40 \mu \mathrm{m}$ and stored in the anti-freeze solution at $-20^{\circ} \mathrm{C}$ for further usage. Control mice showed no red or green fluorescence in any brain regions examined (data not shown). 


\section{Electrophysiological recordings in brain slices}

Surgery-Postnatal day (P)25-P31 Lhx6-cre;Hcrt-eGFP, Lhx6-cre;Gad2-NLS-mCherry or Lhx6-creER ${ }^{T 2}$;Lhx6-GFP mice were anaesthetized with ketamine $\left(50 \mathrm{mg} \mathrm{kg}^{-1}\right)$, dexmedetomidine $\left(25 \mu \mathrm{m} \mathrm{kg}^{-1}\right)$ and the inhalation anaesthetic isoflurane (1-3\%) and fixed in a custom-made stereotaxic frame. A small craniotomy was made and 50-100 $\mathrm{nl}$ of the AAV9-EF1a-DIO-hChR2(H134R)-mCherry vector (University of Pennsylvania Vector Core) was pressure-injected into the zona incerta (AP: $-1.35 \mathrm{~mm}$, ML: $0.3 \mathrm{~mm}$ lateral and DV: $-4.7 \mathrm{~mm}$ ) through a glass pipet (15-20- $\mu \mathrm{m}$ tip diameter, Drummond). For experiments performed with Lhx6-cre;Gad2-NLS-mCherry mice, 50-100 nl of AAV9-EF1a-DIOhChR2(H134R)-eYFP (University of Pennsylvania Vector Core) was injected. The analgesic buprenorphine $\left(0.05 \mathrm{mg} \mathrm{kg}^{-1}\right)$ was administered to all mice post-operatively. For experiments with $L h \times 6-c r e E R^{T 2}$;Lhx6-GFP mice only, beginning on the fifth day following viral injection, $0.5 \mathrm{mg}$ of 4-hydroxytamoxifen dissolved in corn oil was administered by intraperitoneal injection once a day for five days to activate expression of Cre recombinase. Mice were euthanized 11-31 days after virus injection for use in electrophysiological experiments.

Brain slice preparation and cell identification-Mice were anaesthetized with isoflurane and the brains were rapidly removed and chilled in ice-cold sucrose solution containing (in $\mathrm{mM}$ ): $76 \mathrm{NaCl}, 25 \mathrm{NaHCO}_{3}, 25$ glucose, 75 sucrose, $2.5 \mathrm{KCl}, 1.25 \mathrm{NaH}_{2} \mathrm{PO}_{4}$, $0.5 \mathrm{CaCl}_{2}$ and $7 \mathrm{MgSO}_{4}, \mathrm{pH} 7.3$. Acute brain slices $(300 \mu \mathrm{m})$ were prepared in a coronal orientation using a vibratome (VT-1200s, Leica). Slices were then incubated in warm (32$\left.35^{\circ} \mathrm{C}\right)$ sucrose solution for $30 \mathrm{~min}$, then transferred to warm $\left(32-34^{\circ} \mathrm{C}\right)$ artificial cerebrospinal fluid (ACSF) composed of (in $\mathrm{mM}$ ): $125 \mathrm{NaCl}, 26 \mathrm{NaHCO}_{3}, 2.5 \mathrm{KCl}, 1.25$ $\mathrm{NaH}_{2} \mathrm{PO} 4,1 \mathrm{MgSO}_{4}-7 \mathrm{H}_{2} \mathrm{O}, 20 \mathrm{~d}-(+)$-glucose, $2 \mathrm{CaCl}_{2}-2 \mathrm{H}_{2} \mathrm{O}, 0.4$ ascorbic acid, 2 pyruvic

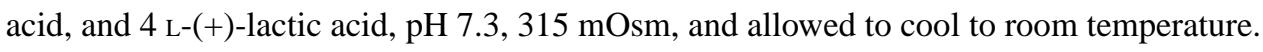
All solutions were continuously bubbled with $95 \% \mathrm{O}_{2} / 5 \% \mathrm{CO}_{2}$.

For whole-cell recordings, slices were transferred to a submersion chamber on an upright microscope (Zeiss AxioExaminer, Objectives: 5×, $0.16 \mathrm{NA}$ and 40×, 1.0 NA) fitted for infrared differential interference contrast (IR-DIC) and fluorescence microscopy. Slices were continuously superfused $\left(2-4 \mathrm{ml} \mathrm{min}^{-1}\right)$ with warm oxygenated ACSF $\left(32-34^{\circ} \mathrm{C}\right)$. Neurons were visualized with a digital camera (Sensicam QE; Cooke) using either transmitted light or epifluorescence. Hcrt neurons in Lhx6-cre;Hcrt-eGFP mice were identified based on their eGFP expression (Extended Data Fig. 5). In Lhx6-cre;Gad2-NLS-mCherry mice, Gad2 neurons were identified based on their mCherry expression (Extended Data Fig. 5). Because Lhx6 neurons express Gad2, the response to $500 \mathrm{~ms}$ of photostimulation with blue light recorded in voltage-clamp was used to eliminate ChR2-expressing Lhx6 neurons from the analysis. In $L h x 6-c r e E R^{T 2}$; Lhx6-eGFP mice, Lhx6 neurons were categorized as either ChR2-expressing Lhx6 cells or untransfected cells based on mCherry expression and the response to $500 \mathrm{~ms}$ of photostimulation with blue light recorded in voltage-clamp (Extended Data Fig. 5).

Whole-cell recordings and analysis-Glass recording electrodes (2-4 M $\Omega$ ) were filled with an internal solution containing (in $\mathrm{mM}$ ): $36.4 \mathrm{KCl}, 96.4 \mathrm{KMeSO}_{3}, 9.1 \mathrm{HEPES}, 0.18$ 
EGTA, 4 MgATP, 0.3 NaGTP, 20 phosphocreatine(Na), pH 7.3, 295 mOsm. Biocytin (0.25\% $\mathrm{w} / \mathrm{v}$ ) was added to the internal solution. Whole-cell patch-clamp recordings were obtained using a Multiclamp 700B amplifier (Molecular Devices), low-pass filtered at $10 \mathrm{kHz}$ and digitized at $20-100 \mathrm{kHz}$ using an ITC-18 (Instrutech) controlled by custom software written in Igor Pro (Wavemetrics). The series resistance averaged $11.8 \pm 5.6 \mathrm{M} \Omega$ (mean \pm s.d.) $(n=$ 89 neurons from 17 mice, all $<30 \mathrm{M} \Omega$ ) and was not compensated. For ChR2 photoactivation, a small spot $\left(\sim 315 \mu \mathrm{m}\right.$ diameter) of blue light $\left(6-600 \mathrm{~mW}\right.$ per $\left.\mathrm{mm}^{2}\right)$ was delivered as previously described using a blue LED ( $470 \mathrm{~nm}$; Luminous $)^{41}$ and focused onto the recorded cell. Neurons firing spontaneous action potentials were hyperpolarized with small current injections ( -10 to $-70 \mathrm{pA}$ ) when measuring postsynaptic responses.

Pharmacological experiments used $5 \mu \mathrm{M}$ 2,3-dioxo-6-nitro-1,2,3,4tetrahydrobenzo[f]quinoxaline-7-sulfonamide disodium salt (NBQX; AMPA receptor antagonist), $5 \mu \mathrm{M}(R S)$-3-(2-carboxypiperazin-4-yl)-propyl-1-phosphonic acid (CPP; NMDA receptor antagonist) and $10 \mu \mathrm{M}$ 6-imino-3-(4-methoxyphenyl)-1(6H)pyridazinebutanoic acid hydrobromide (gabazine; $\mathrm{GABA}_{\mathrm{A}}$ receptor antagonist). To test for monosynaptic connections, the sodium channel blocker, tetrodotoxin (TTX, $1 \mu \mathrm{M}$ ) was first applied to the bath to confirm that recorded postsynaptic responses were action-potential dependent. Then the potassium channel blocker 4-AP $(100 \mu \mathrm{M})$ was added along with TTX to test for monosynaptic connections ${ }^{18}$. All drugs were purchased from Tocris. The resting membrane potential was measured after whole-cell configuration was achieved. The input resistance was determined by measuring the voltage change in response to a 1-s hyperpolarizing current step ( -10 to $-25 \mathrm{pA})$. The amplitude of the sag response was calculated as the difference between the minimum membrane potential following the initiation of a -25-pA current step and the steady-state membrane potential at the end of the 1-s step. Data analysis was performed in Igor Pro (Wavemetrics), Excel (Microsoft), OriginPro 8 (OriginLab) and SigmaPlot (Systat Software). To determine whether Hcrt, Gad2 or Lhx6 neurons in the lateral hypothalamus received Lhx6 input, 20 or more postsynaptic responses to two brief 3-ms photostimulations were first averaged. As a subset of neurons exhibited hyperpolarizing responses shortly after break-in, neurons where the absolute value of the response to the first photostimulation exceeded $0.1 \mathrm{mV}$ and two times the root mean square of the $500 \mathrm{~ms}$ preceding the first photostimulation were identified as responsive neurons (Hcrt neurons: 15 out of 20 neurons recorded from four mice responded with one hyperpolarizing response; Gad2 neurons: 17 out of 31 neurons recorded from five mice responded with five hyperpolarizing responses; Lhx6 neurons: 6 out of 18 recorded neurons recorded from three mice responded with two hyperpolarizing responses). To determine the failure rate, each trace was assessed in a similar manner for a response. For responses in Hcrt and Gad2 neurons, the response latency was measured as the time point at which the rise time exceeded $0.1 \mathrm{mV} \mathrm{ms}^{-1}$ in depolarizing responses, except for two Gad2 neurons for which the response was too small to assess. Data are presented as the mean \pm s.e.m. unless otherwise noted.

Confirmation of the identity of recorded neurons-Brain slices were first fixed in 4\% PFA in 0.01 M PBS after recordings, then slices were incubated with blocking buffer containing 2\% Triton X-100 (Sigma-Aldrich) and either 5\% normal goat serum or 5\% normal donkey serum in PBS for $1 \mathrm{~h}$ at room temperature. To visualize biocytin-filled cells 
and confirm their identity, slices were incubated with Alexa Fluor 350-conjugated streptavidin (1:200, S11249, ThermoFisher Scientific) and either chicken anti-GFP antibody (1:300, GFP-1020, Aves) for Hcrt-GFP neurons or rabbit anti-DsRed antibody $(1: 1,000$, 632496, Clontech) for Gad2-mCherry neurons in PBS containing 1\% Triton X-100 and 5\% corresponding serum overnight. Secondary antibodies used were either Alexa Fluor 488conjugated goat anti-chicken antibody (1:1,000, A11039, ThermoFisher Scientific) or Alexa Fluor 568-conjugated donkey anti-rabbit antibody (1:1,000, A10042, ThermoFisher Scientific). Images were acquired using a Zeiss Cell Observer and Zeiss 510 confocal microscope.

\section{Immunohistochemistry}

Mice were anaesthetized with ketamine and transcardially perfused with $20 \mathrm{ml} 1 \times$ PBS followed by $50 \mathrm{ml}$ of $4 \%$ PFA ( $\mathrm{m} / \mathrm{v})$ in PBS. Brains were removed, fixed overnight in $4 \%$ PFA, followed by $30 \%$ sucrose $(\mathrm{m} / \mathrm{v})$ in PBS overnight in $4^{\circ} \mathrm{C}$ for cryoprotection, and embedded and frozen at $-80^{\circ} \mathrm{C}$. Using a cryostat, the frozen brains were sectioned into $40-$ $\mu \mathrm{m}$ coronal slices for immunohistochemistry. Immunohistochemistry was performed as described previously with minor changes ${ }^{9}$. Brain sections were washed with $1 \times$ PBS and incubated with Superblock (Thermo Scientific, 37515) to block non-specific binding sites. After briefly washing the sections, primary antibodies were diluted in a blocking solution of $5 \%$ horse serum in $0.25 \%$ Triton X-100 in PBS. The following primary antibodies were used: rabbit anti-GFP (1:500, A-6455, Invitrogen); mouse anti-Lhx6 (1:100, sc-271433(Clone A-9), Santa Cruz Biotechnology), goat anti-Hrct (1:400, sc-8070, Santa Cruz Biotechnology), rabbit anti-Fos (1:400, sc-52, Santa Cruz Biotechnology), rabbit antityrosine hydroxylase (TH) (1:400, p4010-150, Pel-Freez), rabbit anti-parvalbumin (1:400, PV 27, Swant) and rabbit anti-GABA (1:200, A2052, Sigma-Aldrich). Brain sections were incubated in primary antibodies and blocking solution overnight at $4^{\circ} \mathrm{C}$.

Secondary antibody in blocking solution was then used to incubate the slides for $2 \mathrm{~h}$ at room temperature. Secondary antibodies used were: Alexa Fluor 488-conjugated donkey antirabbit (1:500, A21206, Invitrogen), Alexa Fluor 488-conjugated donkey anti-mouse (1:500, A212062, Invitrogen), Alexa Fluor 488-conjugated donkey anti-goat (1:500, A11055, Invitrogen), Alexa Fluor 594-conjugated donkey anti-rabbit (1:500, A21207, Invitrogen), Alexa Fluor 594-conjugated donkey anti-mouse (1:500, A21203, Invitrogen) and Alexa Fluor 594-conjugated donkey anti-goat (1:500, A11058, Invitrogen). After washing in $0.1 \%$ Triton X-100 in 1× PBS and staining with DAPI (1:5,000, 10236276001, Roche), brain sections were mounted on positively charged slides with Vectashield Mounting medium (H-1500, Vector laboratories). Fluorescent images were taken using a Zeiss Axioskop 2 Mot Plus Microscope or Zeiss Meta 510 LSM confocal microscope.

For staining of rabies virus-injected brain sections, brain sections were washed twice with TBS, followed by permeabilization with $0.5 \%$ Triton X-100 TBS for 20 min and blocking with $3.5 \%$ donkey serum in TBS $+0.1 \%$ Triton $X-100$ for 30 min. Sections were then incubated in the primary antibody overnight at $4{ }^{\circ} \mathrm{C}$ on the shaker. On the second day, brain sections were washed with TBS and then incubated with secondary antibody at room temperature for $2 \mathrm{~h}$. Primary antibodies and their dilutions are as follows: goat anti-GFP 
(1:500, 600-101-D16, Rockland), rat anti-mCherry (1:500, M11217, Thermo Scientific), mouse anti-Lhx6 (1:400, sc-271433, Santa Cruz), rabbit anti-GABA (1:200, A2052, SigmaAldrich), goat anti-ChAT (1:100, Sigma-Aldrich), rabbit anti-HDC (1:1000, 16045, Progen), rabbit anti-Tph2 (1:400, AB111828, Abcam), rabbit anti-TH (1:200, P40101-150, Pel Freez). Images were then acquired with an Olympus FLUOVIEW1000 confocal microscope using a 10× (NA 0.4 ) or $40 \times$ oil (NA 1.30) lens and further processed by ImageJ (Fig. 1k-t and Extended Data Figs 2, 4).

\section{Fluorescence in situ hybridization (FISH) with immunohistochemistry}

We performed FISH analysis in conjunction with anti-GFP immunohistochemistry on Lhx6eGFP mice with in situ markers including Gad1 and somatostatin (Sst) (Fig. 1 and Extended Data Fig. 1). The RNA probes were generated using the following EST sequences as templates: Gad1 (GenBank accession AI845043), Sst (GenBank accession AI848192). Brains were removed from transgenic Lhx6-eGFP mice within 5 min after euthanasia and fixed overnight in $4 \%$ PFA $(\mathrm{m} / \mathrm{v})$ in $1 \times \mathrm{PBS}$ at $4^{\circ} \mathrm{C}$ followed by incubation in $30 \%$ sucrose $(\mathrm{m} / \mathrm{v})$ in PBS overnight in $4^{\circ} \mathrm{C}$ for cryoprotection. The embedded and frozen brains were sectioned into $40-\mu \mathrm{m}$ sections using a cryostat and then mounted onto positively charged slides. For FISH, all equipment was cleaned with $0.3 \mathrm{M}$ sodium hydroxide and RNaseZap and all solutions were DEPC-treated and autoclaved.

FISH was carried out as previously described ${ }^{42}$ with some alterations. In brief, slides were fixed in $4 \%$ PFS $(\mathrm{m} / \mathrm{v})$ in $1 \times$ PBS for $15 \mathrm{~min}$ and then cell membranes were digested by incubation in $1 \mu \mathrm{g} \mathrm{ml}^{-1}$ proteinase $\mathrm{K}$ with $50 \mathrm{mM}$ Tris $\mathrm{pH} 7.5$ and $5 \mathrm{mM}$ EDTA in $1 \times$ PBS for $15 \mathrm{~min}$. After re-fixation in 4\% PFA, the brain sections underwent acetylation with $0.27 \%$ acetic anhydride and $10 \% 1 \mathrm{M}$ TEA $(\mathrm{pH} 8)$ in $1 \times$ PBS. The slides were then washed in $1 \times$ PBS, incubated in $0.1 \%$ Triton X-100 in PBS for $1 \mathrm{~h}$ and then quenched in $1 \%$ hydrogen peroxide in PBS for 30 min to eliminate endogenous peroxidase activity. Next, the slides were incubated in hybridization buffer (50\% formamide, $5 \times$ saline sodium citrate (SSC)) for $2 \mathrm{~h}$ and incubated overnight under siliconized coverslips in heat-activated DIGconjugated RNA probes diluted in hybridization buffer at $68^{\circ} \mathrm{C}$. The next day, a $5 \times \mathrm{SSC}$ wash was followed by two $0.2 \times \mathrm{SSC}$ washes to remove the coverslips and to eliminate nonspecific probe binding. The slides were then washed with $0.3 \%$ Triton X-100 in PBS and incubated in blocking buffer (0.1\% Triton X-100, 1\% HISS in $1 \times$ PBS) for $1 \mathrm{~h}$.

For detection of Lhx $6^{+}$neurons, immunohistochemistry for anti-eGFP was combined with the subsequent in situ hybridization steps. The slides were incubated overnight at $4{ }^{\circ} \mathrm{C}$ in a blocking solution containing rabbit anti-GFP antibody (1:500, A-6455, Invitrogen) and antidigoxigenin-POD antibody $(1: 1,000,1207733$, Roche). After washing the slides in $0.3 \%$ Triton X-100 multiple times on the third day, the probe signal was recovered by Cy3tyramide amplification reagent (1:125, NEL752001KT, TSA Plus cyanine 3/cyanine 5 system, PerkinElmer) in $1 \times$ amplification diluent and monitored frequently by fluorescent microscopy for the timing of signal appearance. Slides were washed, quenched in $1 \%$ hydrogen peroxide and $0.3 \%$ Triton X-100 in PBS and blocked in blocking solution $(0.2 \%$ Triton X-100, $1 \%$ horse serum, $0.1 \%$ BSA powder) for $1 \mathrm{~h}$. Primary antibody signal was then detected by species-specific Alexa Fluor 488-conjugated donkey anti-rabbit (1:500; 
A21206, Invitrogen) incubated with blocking solution for $2 \mathrm{~h}$. Slides were then washed and coverslipped in Vectashield. Fluorescence images were taken using a Zeiss Axioskop 2 Mot Plus Microscope or Zeiss Meta 510 LSM confocal microscope.

\section{Statistics}

The experiments were not randomized. No statistical methods were used to predetermine sample size. For Fos immunohistochemistry analysis, each group consisted of data obtained from four mice, a total of four bilateral sections containing the zona incerta was analysed for each mouse, which resulted in interdependence in each group. As a result, to analyse this data, we applied a linear mixed-effect model fit by REML, a non-parametric method, which can resolve issues of interdependence in the Fos data. A two-tailed, one-way ANOVA was used to evaluate the differences among multiple groups. A two-way ANOVA with Bonferonni correction was used to assess pharmacological manipulations in electrophysiological experiments. All sleep-wake data analysis was analysed by two-way ANOVA followed by Sidak's post hoc test, and later confirmed by a non-parametric method, Wilcoxon/Mann-Whitney $U$-test. Statistical analysis was conducted using the statistical programming language R (R Foundation for Statistical Computing) and Excel (Microsoft).

\section{Data availability}

Source Data for all figures are provided with the online version of the paper. 


\section{Extended Data}
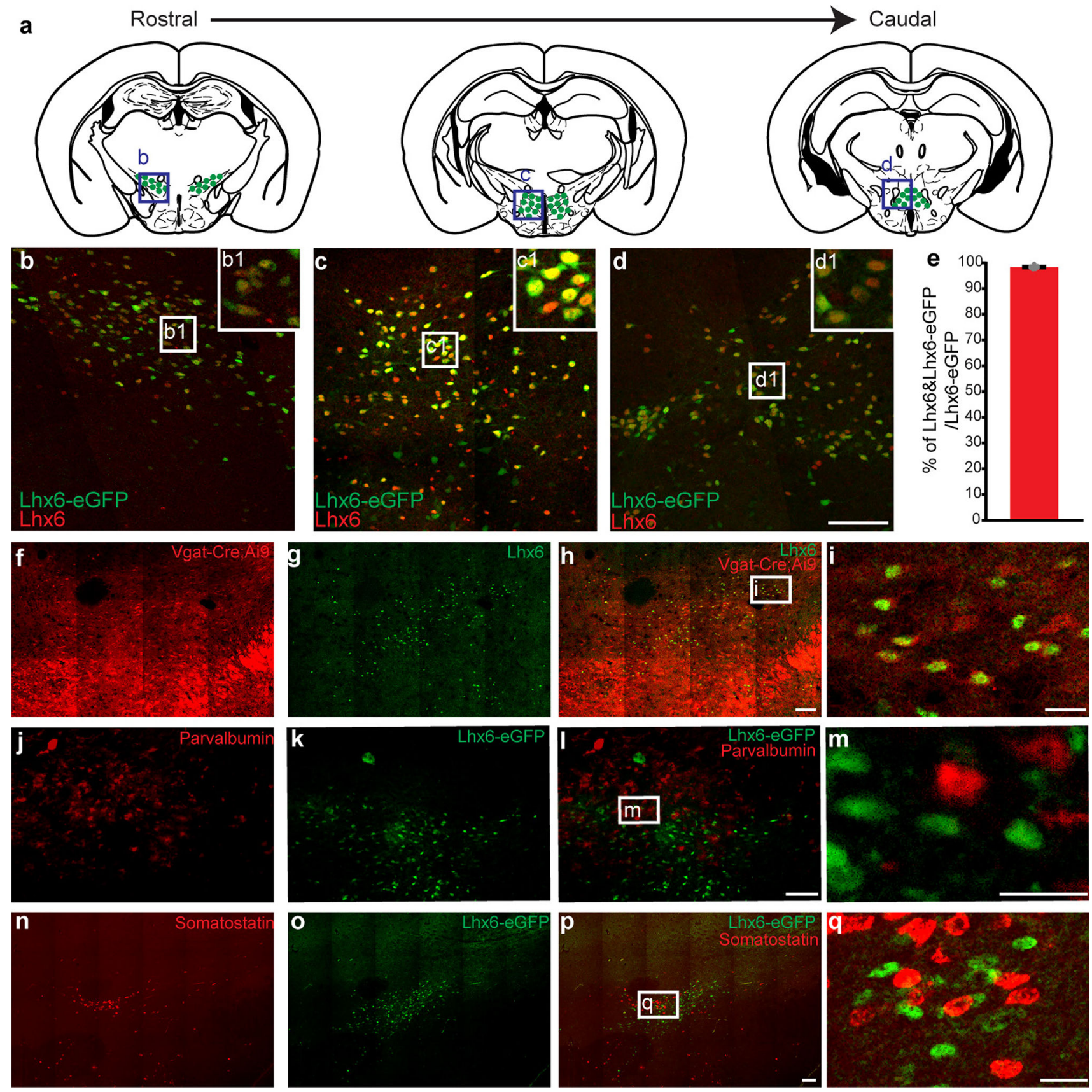

Extended Data Figure 1. Distribution of diencephalic $\mathrm{Lhx6}^{+}$neurons and immunohistochemical analysis of Lhx6-expressing neurons

Continued from Fig. 1. a, Schematics showing the distribution of Lhx $6^{+}$neurons in diencephalon. Blue boxes indicate $\mathrm{Lhx} 6^{+}$neurons (green) in the zona incerta, dorsomedial hypothalamus and the posterior hypothalamus, with representative images shown in b-d. bd, Co-expression of eGFP (green) and Lhx6 in Lhx6-eGFP line (red) in zona incerta (b), dorsomedial hypothalamic nucleus (c), and posterior hypothalamus (d). eGFP signal fills the cell cytoplasm, whereas Lhx6 immunostaining is nuclear. Insets show magnified images of 
b-d. Image in b is identical to main Fig. $1 \mathrm{~b}$ and is included for comparison. Scale bar, 100 $\mu \mathrm{m}$. e, The percentage Lhx6 ${ }^{+}$and eGFP-expressing neurons in the Lhx6-eGFP line over the number of Lhx6-eGFP ${ }^{+}$neurons in the zona incerta. $n=5$ mice, eight sections per group. Data are mean \pm s.e.m. f-i, Representative images showing Vgat-Cre;Ai9 (f, red) and Lhx6 ${ }^{+}$ neurons ( $\mathbf{g}$, green) in the zona incerta. Merged image is shown in $\mathbf{h}$ and magnification is shown in $\mathbf{i}$. $\mathbf{j}-\mathbf{m}$, Representative images showing Pvalb ${ }^{+}$neurons $\left(\mathbf{j}\right.$, red) and Lhx6-eGFP ${ }^{+}$ neurons (k, green) in the zona incerta. Merged image is shown in $\mathbf{I}$ and magnification is shown in $\mathbf{m}$. n-q, Representative images showing somatostatin neurons from FISH (n, red) and Lhx6-eGFP neurons (o, green) in the zona incerta. Merged image is shown in $\mathbf{p}$ and magnification is shown in $\mathbf{q}$. Scale bars, $100 \mu \mathrm{m}(\mathbf{d}, \mathbf{f}-\mathbf{h}, \mathbf{j}-\mathbf{l}, \mathbf{n}-\mathbf{p})$ and $25 \mu \mathrm{m}(\mathbf{i}, \mathbf{m}, \mathbf{q})$. 

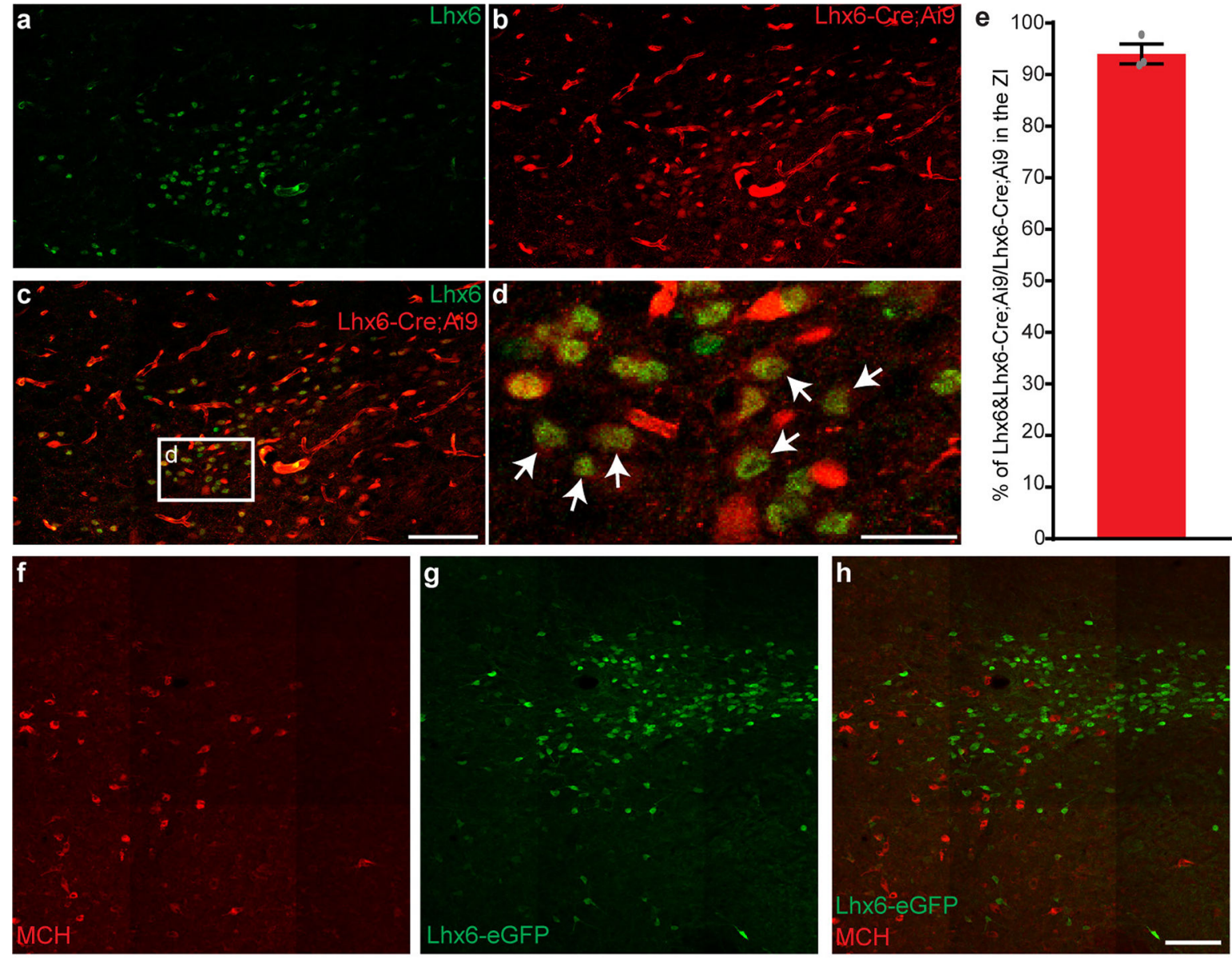

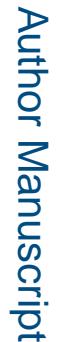
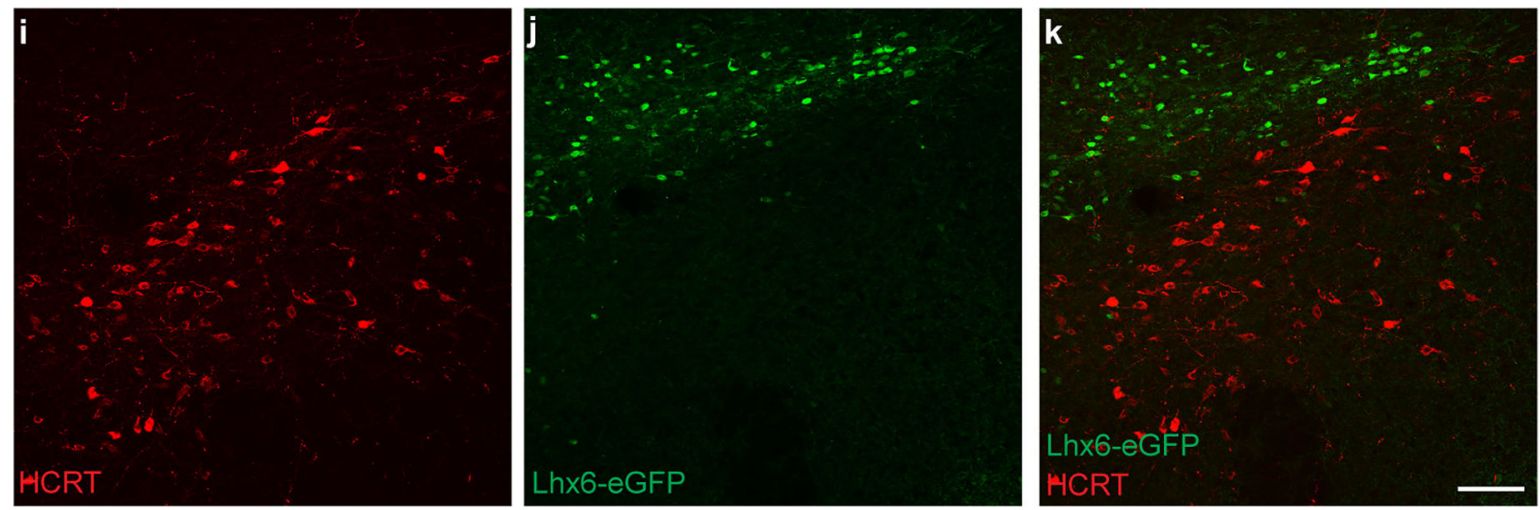

Extended Data Figure 2. Lhx6-Cre activity recapitulates endogenous Lhx6 expression pattern and immunohistochemical analysis of Lhx6-expressing neurons

Continued from Extended Data Fig. 1. a-d, Representative images showing Lhx6 $6^{+}$neurons in the zona incerta (a, green), tdTomato expression in the Lhx6-cre;Ai9line (b, red). tdTomato staining is also observed in endothelial cells, where Lhx6 expression is not detected in adult mouse brains. Merged image is shown in $\mathbf{c}$ and magnification is shown in d. Arrows indicate examples of colocalization of Lhx6 and tdTomato expression. e, The percentage $\mathrm{Lhx}^{+}{ }^{+}$ddTomato $^{+}$neurons relative to tdTomato ${ }^{+}$neurons in the zona incerta in the Lhx6-cre;Ai9 line. $n=3$ mice. Data are mean \pm s.e.m. f-h, Representative images showing 
$\mathrm{MCH}^{+}$neurons (f, red) in the lateral hypothalamus and Lhx6-eGFP neurons (g, green) in the zona incerta. Merged image is shown in $\mathbf{h}$. $\mathbf{i}-\mathbf{k}$, Representative images showing $\mathrm{Hcrt}^{+}$ neurons (i, red) in the lateral hypothalamus and Lhx6-eGFP neurons ( $\mathbf{j}$, green) in the zona incerta. Merged image is shown in $\mathbf{k}$. Scale bars, $100 \mu \mathrm{m}(\mathbf{a}-\mathbf{c}, \mathbf{f}-\mathbf{k})$ and $25 \mu \mathrm{m}(\mathbf{d})$.
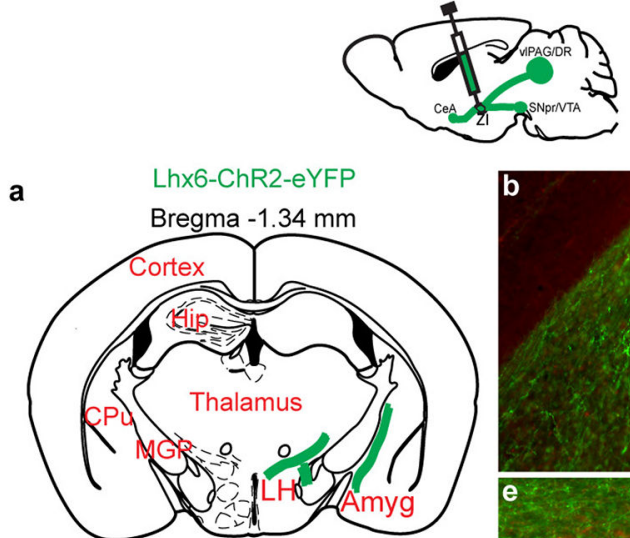

Bregma $-2.30 \mathrm{~mm}$

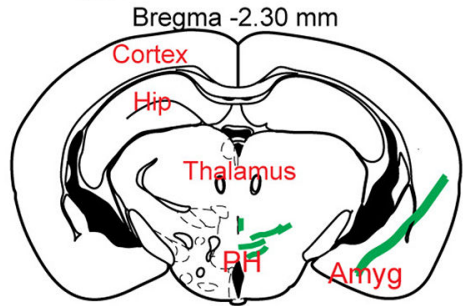

Bregma $-2.92 \mathrm{~mm}$
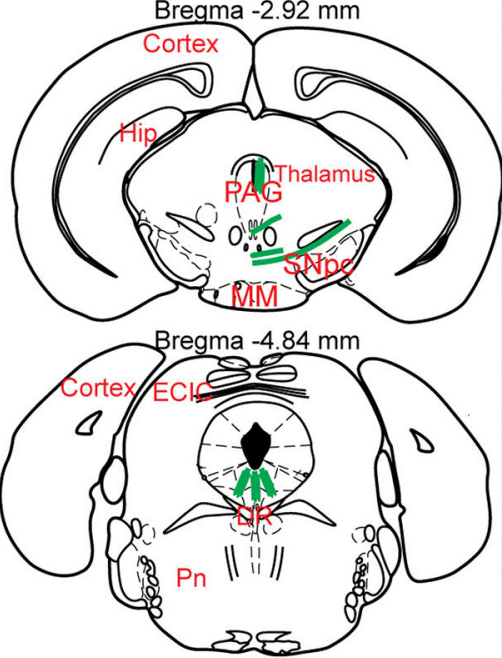

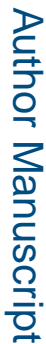
zona incerta
Allen Brain Atlas Mouse Connectome

Slc32a1(Vgat)-IRES-Cre
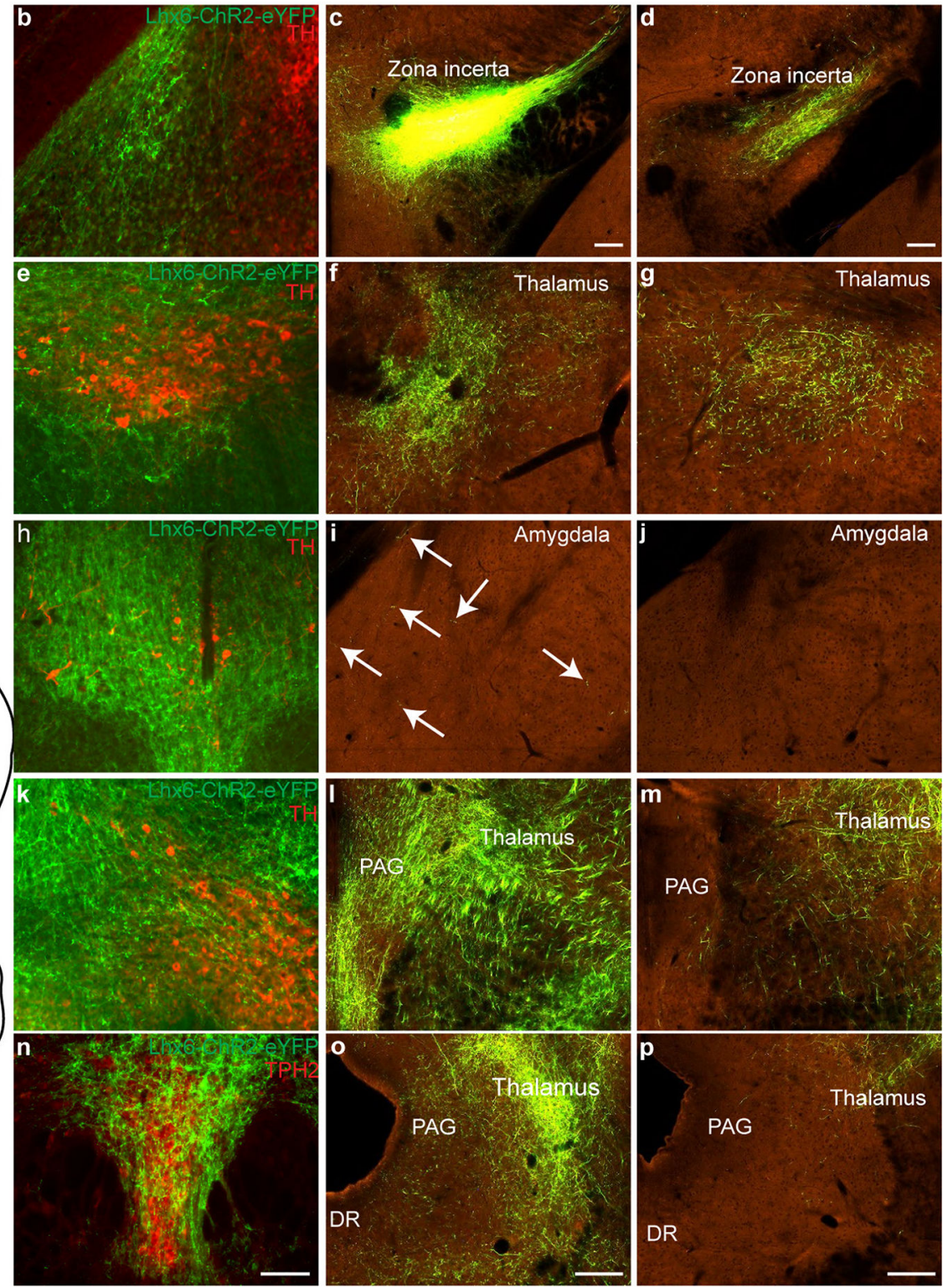

Extended Data Figure 3. Additional data showing mapping of neural projection sites of Lhx6expressing neurons in the VZI, and projection sites of SIc32a1 and Pvalb-expressing cells in the

Continued from Fig. 1. a, Schematics showing neural projection sites of $\operatorname{Lhx} 6^{+}$neurons in the zona incerta across multiple brain regions after ChR2-eYFP virus injection into the zona 
incerta of the Lhx6-cre line. $\mathbf{b}, \mathbf{e}, \mathbf{h}, \mathbf{k}, \mathbf{n}$, Representative images of eYFP ${ }^{+}$axonal processes of Lhx6 $6^{+}$neurons of the VZI (green) in amygdala (b), substantia nigra (e), periaqueductal grey (h), ventral tegmental area (k) and dorsal raphe nucleus (n). TH (red) immunostaining was used to demarcate the amygdala, substantia nigra, periaqueductal grey and ventral tegmental area in $\mathbf{b}, \mathbf{e}, \mathbf{h}$ and $\mathbf{k}$. TPH2 (red) immunostaining was used to demarcate the dorsal raphe nucleus in $\mathbf{n}$. Scale bars, $100 \mu \mathrm{m}$. c, f, $\mathbf{i}, \mathbf{l}, \mathbf{o}$, High-power images of the brain of a SIc32a1-cre mouse injected with DIO-rAAV-eGFP in the zona incerta, demonstrating infection in zona incerta (c), central thalamus (f, o), amygdala (i) and periaqueductal grey (l). White arrows indicate eGFP ${ }^{+}$processes in i. Scale bars, $200 \mu \mathrm{m}$. d, g, j, m, p, Highpower images of the brain of a Pvalb-cre mouse injected with DIO-rAAV-eGFP in zona incerta. $\mathrm{GFP}^{+}$processes are detected in the zona incerta (d) and central thalamus $(\mathbf{g}, \mathbf{m})$. No signal is detected in the amygdala $(\mathbf{j})$ and very little signal is seen in the periaqueductal grey (m). Scale bars, $200 \mu \mathrm{m} . \mathbf{c}, \mathbf{d}, \mathbf{f}, \mathbf{g}, \mathbf{i}, \mathbf{j}, \mathbf{l}, \mathbf{m}, \mathbf{o}, \mathbf{p}$, Images were obtained from the Allen Brain Atlas Mouse Connectome Project. Experiment numbers 17106613 for Scl32a1-IRES-Cre (section 73 (c, f, i), section 58 (l) and section 44 (o)) and 301539438 for Pvalb-IRES-Cre (section $68(\mathbf{d}, \mathbf{g}, \mathbf{j})$, section $60(\mathbf{m})$ and section $45(\mathbf{p})$ ). Amyg, amygdala; $\mathrm{CPu}$, caudate putamen; DR, dorsal raphe nucleus; ECIC, external cortex of the inferior colliculus; Hip, hippocampus; LH, lateral hypothalamus; MGP, medial globus pallidus; MM, mammillary nucleus; PAG, periaqueductal grey; PH, posterior hypothalamus; Pn, paranigral nucleus; SNpc, substantia nigra pars compact; VTA, ventral tegmental area. 

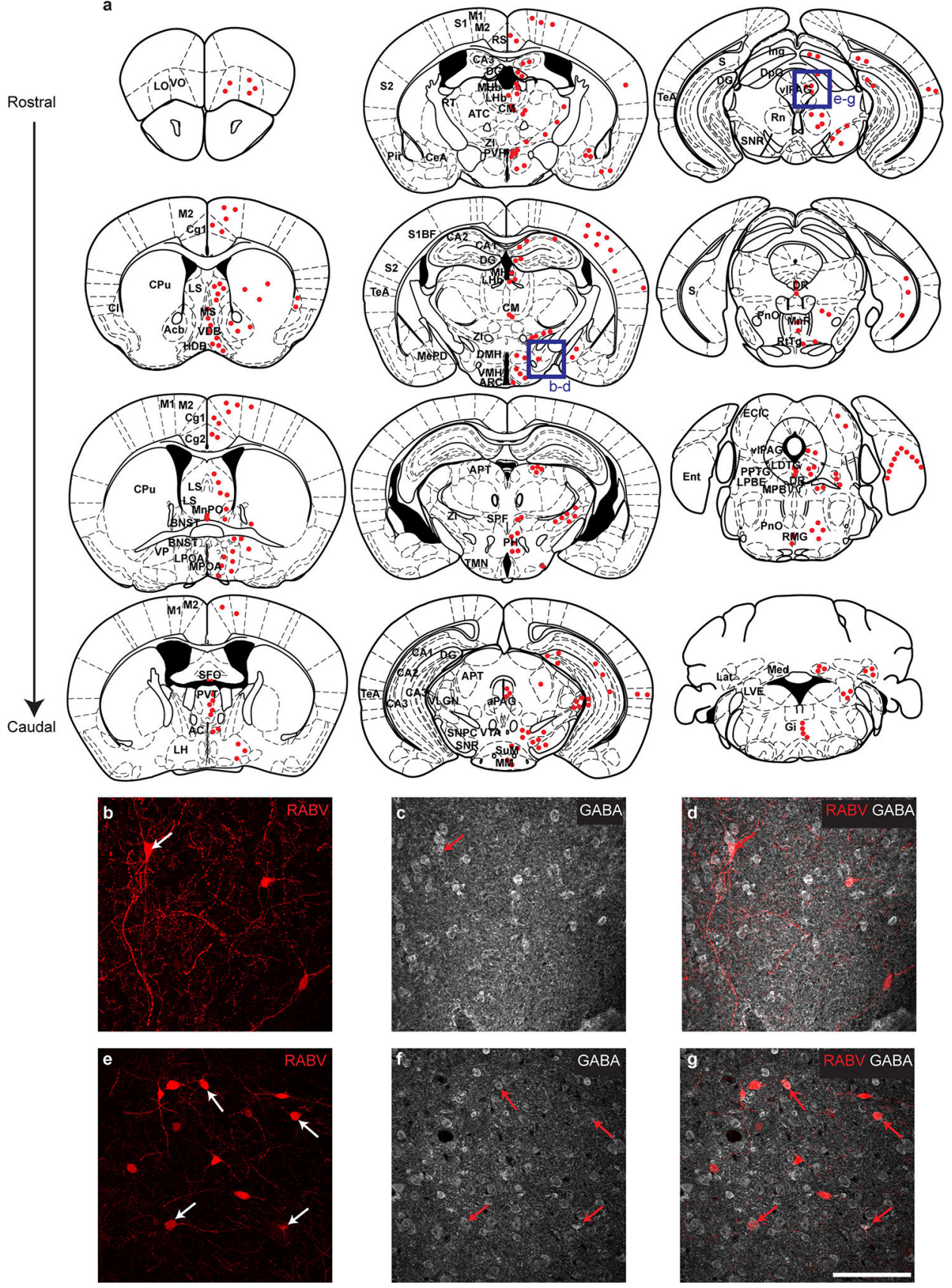

Extended Data Figure 4. Presynaptic inputs to $\mathrm{Lhx6}^{+}$zona incerta neurons

Continued from Fig. 1. a, Schematic showing the distribution of presynaptic neurons (red dots) to Lhx6 zona incerta neurons and location of presynaptic neurons across the rostrocaudal gradient of the mouse brain. For mice injected with rabies + TVA (tumour virus A, the receptor for modified rabies virus), $n=4$. For rabies only controls, $n=2$. b-d, Distribution of presynaptic neurons to $\operatorname{Lhx} 6^{+}$zona incerta neurons located in the lateral hypothalamus (RABV, $\mathbf{b}$, red). GABA immunostaining (c, grey) was used to label neurons in the lateral hypothalamus. Merged image is shown in $\mathbf{d . ~ e - g}$, Distribution of presynaptic neurons to Lhx6 zona incerta neurons located in the periaqueductal grey (RABV, e, red). 
GABA immunostaining (f, grey) was used to label neurons in the periaqueductal grey. Merged is shown in g. Arrows indicate neurons with RABV and GABA co-localization (bg). Scale bar, $100 \mu \mathrm{m}$. AC, anterior commissural nucleus; AR, arcuate nucleus; Acb, nucleus accumbens; aPAG, anterior periaqueductal grey; APT, anterior pretectal nucleus; ATC, anteromedial thalamic nucleus; BNST, bed nucleus of the stria terminalis; CA1, hippocampus CA1; CA3, hippocampus CA3; CeA, central nucleus of the amygdala; $\mathrm{Cg} 1$ / $\mathrm{Cg}$ 2, cingulate cortex; $\mathrm{Cl}$, Claustrum; $\mathrm{CM}$, central medial nucleus of thalamus; $\mathrm{CPu}$, striatum; DG, dentate gyrus; DMH, dorsomedial hypothalamus; DpG, motor-related nuclei of superior colliculus; DR, dorsal raphe nucleus; ECIC, external cortex of the inferior colliculus; Ent, entorhinal cortex; Gi, midline gigantocellular nucleus of the medulla; HDB/ VDB, nucleus of the diagonal band; Ing, intermediate grey layer of superior colliculus; Lat, lateral/dentate cerebellar nucleus; LDTG, laterodorsal tegmental nucleus; LH, lateral hypothalamus; LHb, lateral habenula; LO, lateral orbital cortex; LPBE, lateral parabrachial nucleus of the pons; LPOA, lateral preoptic area; LS, lateral septum; LVE, lateral vestibular nucleus; M1/M2, frontal/secondary motor cortex; Med, fastigial nucleus; MePD, posterodorsal medial amygdaloid nucleus; $\mathrm{MHb}$, medial habenula; $\mathrm{MM}$, medial mammillary nucleus; MnPO, median preoptic nucleus; MnR, median raphe nucleus; MPB, medial parabrachial nucleus; MPOA, medial preoptic area; MS, medial septal nucleus; $\mathrm{PH}$, posterior hypothalamus; Pir, piriform cortex; PnO, pontine reticular nucleus, oral part; PPTG, pedunculopontine tegmental nucleus; PVH, paraventricular nucleus of the hypothalamus; PVT, paraventricular nucleus of thalamus; RMG, nucleus raphe magnus; Rn, red nucleus; RS, retrosplenial area; RT, reticular nucleus; RtTg, midbrain reticular formation; S, subiculum; S1/S2, somatosensory cortex; S1BF, barrel cortex; SFO, subfornical organ; SNPC, substantia nigra pars compact; SNR, substantia nigra, reticular part; SPF, parafascicular thalamic nucleus; SuM, supramammillary nucleus; TeA, temporal association cortex; TMN, tuberomammillary nucleus; VLGN, ventrolateral geniculate nucleus; vlPAG, ventrolateral Periaqueductal grey; VMH, ventromedial hypothalamus; VO, ventral orbital cortex; VP, ventral pallidum; VTA, ventral tegmental area; ZI, zona incerta. 

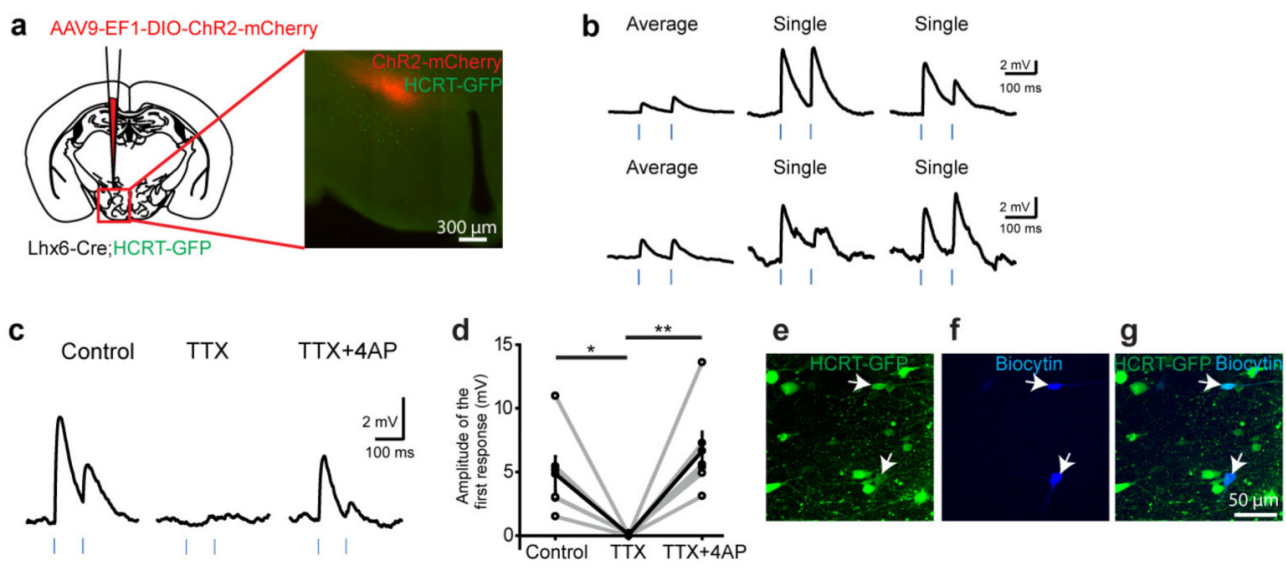

h AAV9-EF1-DIO-ChR2-eYFP

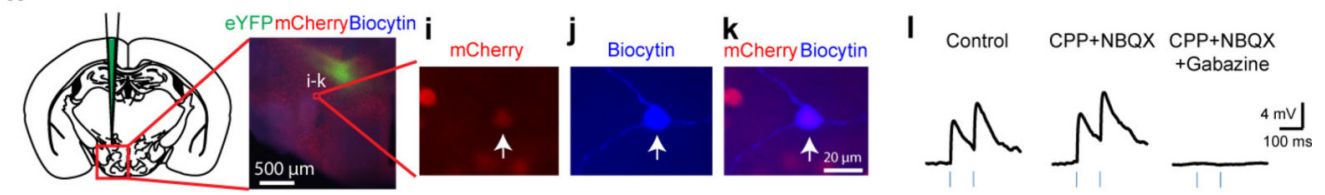

Lhx6-Cre;Gad2-NLS-mCherry

m AAV9-EF1-DIO-ChR2-mCherry
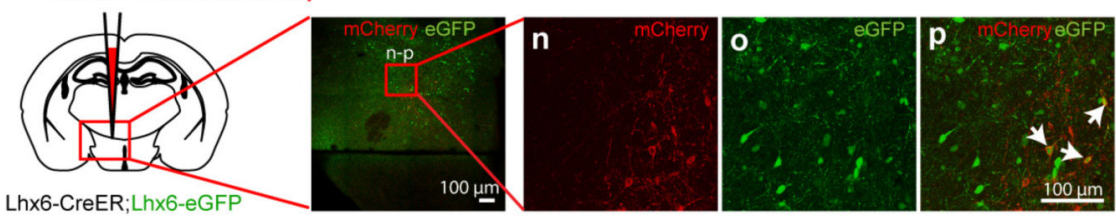

q

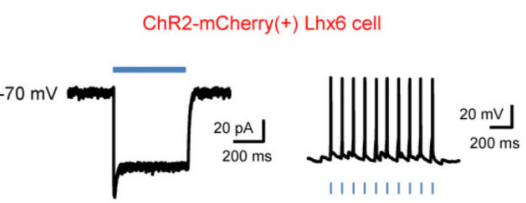

r

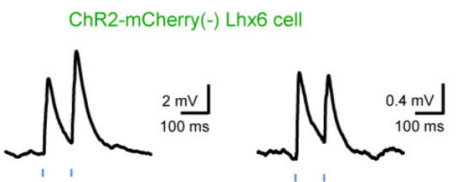

$\mathbf{S}$
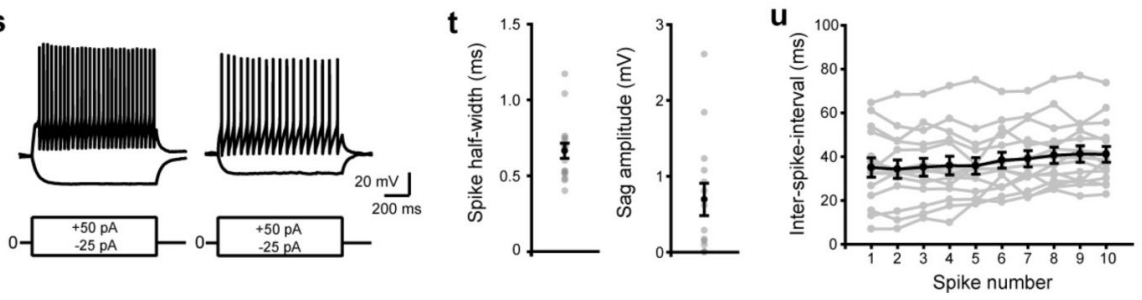

Extended Data Figure 5. Synaptic outputs of Lhx6 neurons in the zona incerta Continued from Fig. 2. a, Schematic showing injection of AAV9-DIO-ChR2-mCherry into the zona incerta of Lhx6-cre;Hcrt-eGFP mice. The red box shows the location of Hcrtexpressing neurons (green) in the lateral hypothalamus and the ventromedial portion of the zona incerta with $\mathrm{ChR} 2$-expressing $\mathrm{Lhx}^{+}$neurons (red) shown in the image on the right. Scale bar, $300 \mu \mathrm{m}$. b. Responses of two Hcrt neurons to brief ( $3 \mathrm{~ms}$ ) photostimulation of ChR2-expressing Lhx6 axons. The average responses (left) and two representative responses (right) are shown for each neuron. The responses are depolarizing owing to the high chloride internal solution used. The amplitude of the average responses was smaller than for 
individual responses because of the failure rate to any individual photostimulation (24.0 $\pm 5.9 \%$, range $65.8-0 \% ; n=15$ cells). c, Average responses recorded from a Hcrt neuron to photostimulation of ChR2-expressing Lhx6 axons under control conditions (left), following bath application of the sodium channel blocker tetrodotoxin (TTX, middle) and following the addition of the potassium channel blocker 4-AP (right). Note that the cell shows a depolarizing response owing to the high chloride internal recording solution. d, Summary data showing the amplitude of the average response to the first photostimulation under control conditions, following bath application of TTX and TTX together with 4-AP. The responses recorded using a high chloride internal solution in TTX and 4-AP treatment indicate that ChR2-expressing Lhx6 $6^{+}$VZI neurons form monosynaptic connections onto the recorded $\mathrm{Hcrt}^{+}$neurons (control, $4.85 \pm 1.37 \mathrm{mV}$; TTX, $0.07 \pm 0.04 \mathrm{mV}$; TTX + 4-AP, 6.65 $\pm 1.50 \mathrm{mV}$; two-way ANOVA with Bonferroni correction, control versus TTX, $* P=$ 0.01498 ; TTX versus TTX +4 -AP, $* * P=0.00178$, control versus TTX +4 -AP, $P=0.61814$; $n=6$ cells from four mice). e-g, Expression of eGFP (e, green) in recorded Hcrt-eGFP neurons was confirmed by filling the recorded cells in the lateral hypothalamus with biocytin (f, blue). Arrows indicate colocalization of the two markers in the recorded neurons as seen in the merged image $(\mathbf{g})$. Scale bar, $50 \mu \mathrm{m}$. $\mathbf{h}$, Schematic showing injection of AAV9-DIOChR2-eYFP into the zona incerta of Lhx6-Cre;Gad2-NLS-mCherry mice (left). The red box shows the location of the image (right) showing Gad2-expressing neurons (red) in the lateral hypothalamus and $\mathrm{Lhx}^{+}$neurons (green) in the ventromedial portion of the zona incerta. Scale bar, $500 \mu \mathrm{m}$. i-k, Colocalization of mCherry (i, red) in Gad2-NLS-mCherry mice with biocytin ( $\mathbf{j}$, blue) in $\mathrm{Gad} 2^{+}$neurons recorded in the lateral hypothalamus. The arrow indicates colocalization of the two markers as seen in the merged image (k). Scale bar, $20 \mu \mathrm{m}$. l, Average responses recorded from a Gad2-expressing neuron to brief ( $3 \mathrm{~ms}$ ) photostimulation of ChR2-expressing Lhx6 axons under control conditions (left), following bath application of the ionotropic glutamate receptor antagonists, CPP and NBQX (middle) and following the addition of the $\mathrm{GABA}_{\mathrm{A}}$ receptor antagonist, gabazine (right). Note that the response is depolarizing owing to the high concentration of chloride in the internal recording solution. Responses were eliminated in the presence of gabazine (control, $3.96 \pm 1.55 \mathrm{mV}$; $\mathrm{CPP}+\mathrm{NBQX}, 3.48 \pm 2.16 \mathrm{mV}$; CPP + NBQX + gabazine: $0.01 \pm 0.01 \mathrm{mV} ; n=3$ cells from two mice). $\mathbf{m}$, Schematic showing injection of AAV9-DIO-ChR2-mCherry virus into the zona incerta of $L h \times 6-c r e E R^{T 2}$;Lhx6-eGFP mice (left). The red box indicates the location of a representative image from one mouse (right) showing mCherry-expressing (red) and eGFP-expressing (green) Lhx $6^{+}$neurons in the zona incerta. The red box indicates the location of higher magnification images in $\mathbf{n}-\mathbf{p}$. Scale bar, $100 \mu \mathrm{m}$. n-p, Expression of mCherry $\left(\mathbf{n}\right.$, red) and eGFP (o, green) in $L h \times 6-c r e E R^{T 2} ; L h \times 6-e G F P$ mice injected with AAV9-DIO-ChR2-mCherry in the zona incerta. The merged image is shown in p. Arrows in p indicate a subset of Lhx $6^{+}$neurons that express both ChR2-mCherry and eGFP. Scale bar, $100 \mu \mathrm{m}$. q, Average response elicited by $500 \mathrm{~ms}$ photostimulation in a ChR2-expressing Lhx $6^{+}$VZI neuron recorded in whole-cell voltage-clamp mode (left) and a representative trace of action potentials elicited by brief 3-ms flashes of blue light $(10 \mathrm{~Hz})$ recorded in current-clamp in the same neuron (right). Note that the train of light pulses reliably elicits action potentials. Responses to 500-ms long light pulses recorded in voltage-clamp were used to distinguish ChR2-expressing from non-ChR2-expressing Lhx6 $6^{+}$VZI neurons. $\mathbf{r}$, Representative average responses of two non-ChR2-expressing $\operatorname{Lhx} 6^{+}$neurons to 
photostimulation of axons of ChR2-expressing Lhx $6^{+}$neurons. The responses are depolarizing because of the high concentration of chloride in the internal recording solution. $\mathbf{s}$, Representative responses from two Lhx $6^{+}$neurons in response to steps of depolarizing $(+50 \mathrm{pA})$ current. Average responses to hyperpolarizing current steps $(-25 \mathrm{pA})$ are also shown. $\mathbf{t}$, The spike half-width (left; $n=17$ cells) and sag amplitude (right; $n=14$ cells) of recorded Lhx $6^{+}$neurons. The mean \pm s.e.m. of the responses are shown in black. u, Plot of the inter-spike intervals for trains of action potentials elicited in Lhx $6^{+}$neurons by 1-s steps of depolarizing current. The mean \pm s.e.m. of the responses are shown in black ( $n=15$ cells). 


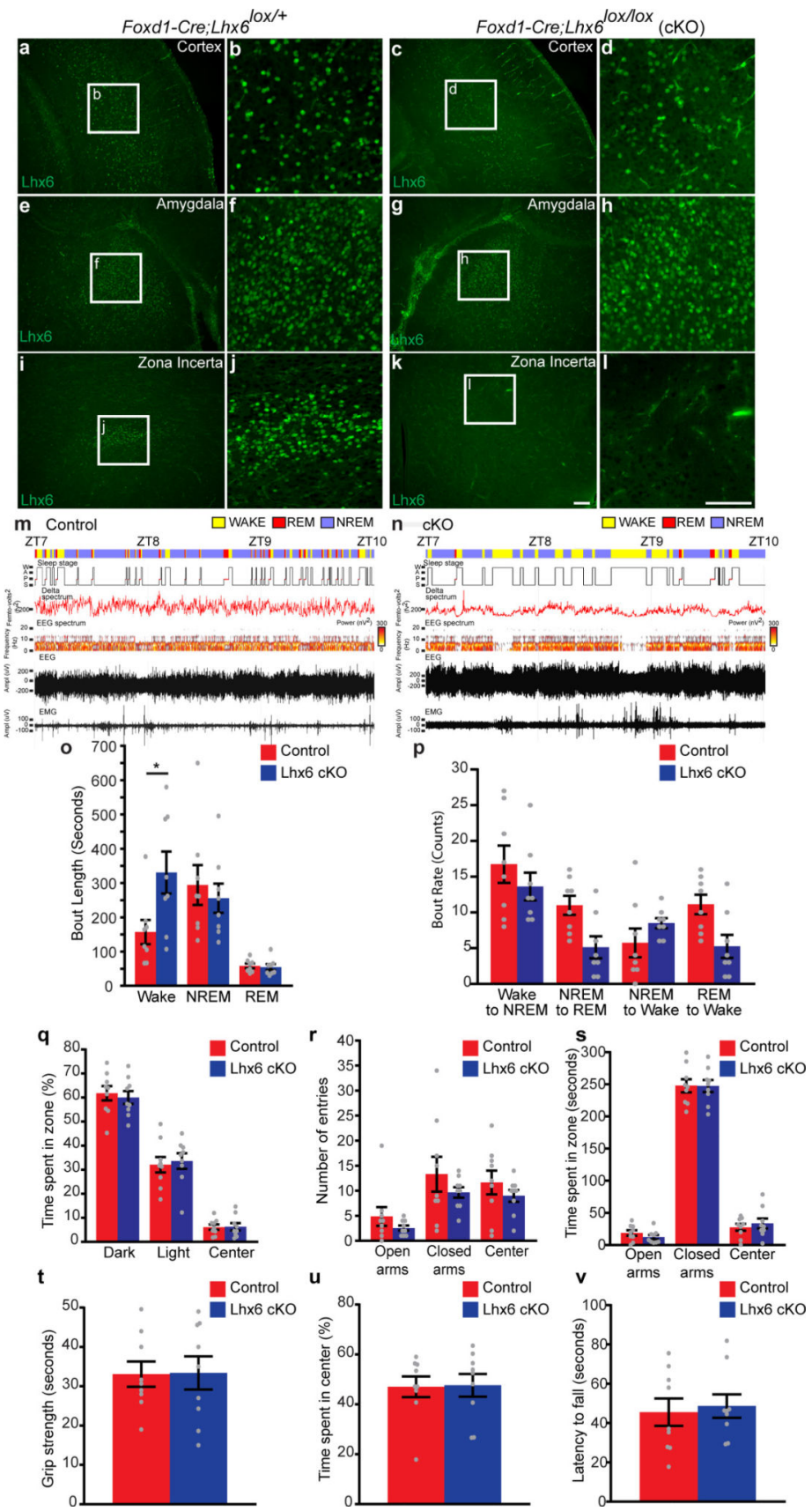

Extended Data Figure 6. Lhx6 expression is selectively deleted in the diencephalon, but preserved in the telencephalon of Lhx6-conditional knockout Foxd1-cre; Lhx ${ }^{\text {lox/lox }}$ (cKO) line, and Lhx6 cKO mice do not display obvious behavioural abnormalities other than changes in sleep patterns

Continued from Fig. 3. a-l, Representative images of Lhx6 immunostaining (green) in the cortex (a-d), amygdala (e-h) and zona incerta (i-l) of control $(\mathbf{a}, \mathbf{b}, \mathbf{e}, \mathbf{f}, \mathbf{i}, \mathbf{j})$ and cKO (c, d, $\mathbf{g}, \mathbf{h}, \mathbf{k}, \mathbf{l})$ mice. Magnified images of $\mathbf{a}, \mathbf{c}, \mathbf{e}, \mathbf{g}, \mathbf{i}, \mathbf{k}$ are shown in $\mathbf{b}, \mathbf{d}, \mathbf{f}, \mathbf{h}, \mathbf{j}, \mathbf{l}$, respectively. Note an absence of Lhx6 expression in the zona incerta of the cKO group. Scale bars, 100 $\mu \mathrm{m} . \mathbf{m}, \mathbf{n}$, Sample EEG recordings for control (m) and cKO (n) mice with wake (yellow), REM sleep (red) and NREM sleep (purple) indicated, along with hypnogram (sleep stage), 
FFT-derived delta power (femtovolts ${ }^{2}$ ), EEG spectrum (frequency), EEG raw activity (amplitude) and EMG raw activity (amplitude) between ZT7 and ZT10. o, p, Bout length quantification (o) showing the duration of wake, NREM and REM episodes and bout count quantification (p) showing sleep-wake transitions in control (Foxd1-cre;Lhx $\sigma^{\text {lox/+ }}$ ) and cKO (Foxd1-cre;Lhx $b^{\text {lox/lox }}$ ) groups. Two-way ANOVA followed by Sidak's post hoc test. $n=8$ control and 8 cKO mice. q-v, Light-dark preference test (q), EPM (r, s), grip strength (t), open field test (u) and rotarod (v) were conducted in control (red) and Lhx6 cKO (blue) groups. $* P<0.05$ for changes in sleep patterns $(\mathbf{m}-\mathbf{p})$; no significant difference $(P>0.1)$ was detected for all other behaviours $(\mathbf{q}-\mathbf{v})$ analysed; two-way ANOVA followed by Sidak's post hoc test. Data are mean \pm s.e.m. $n=8$ control and $8 \mathrm{cKO}$ mice. 

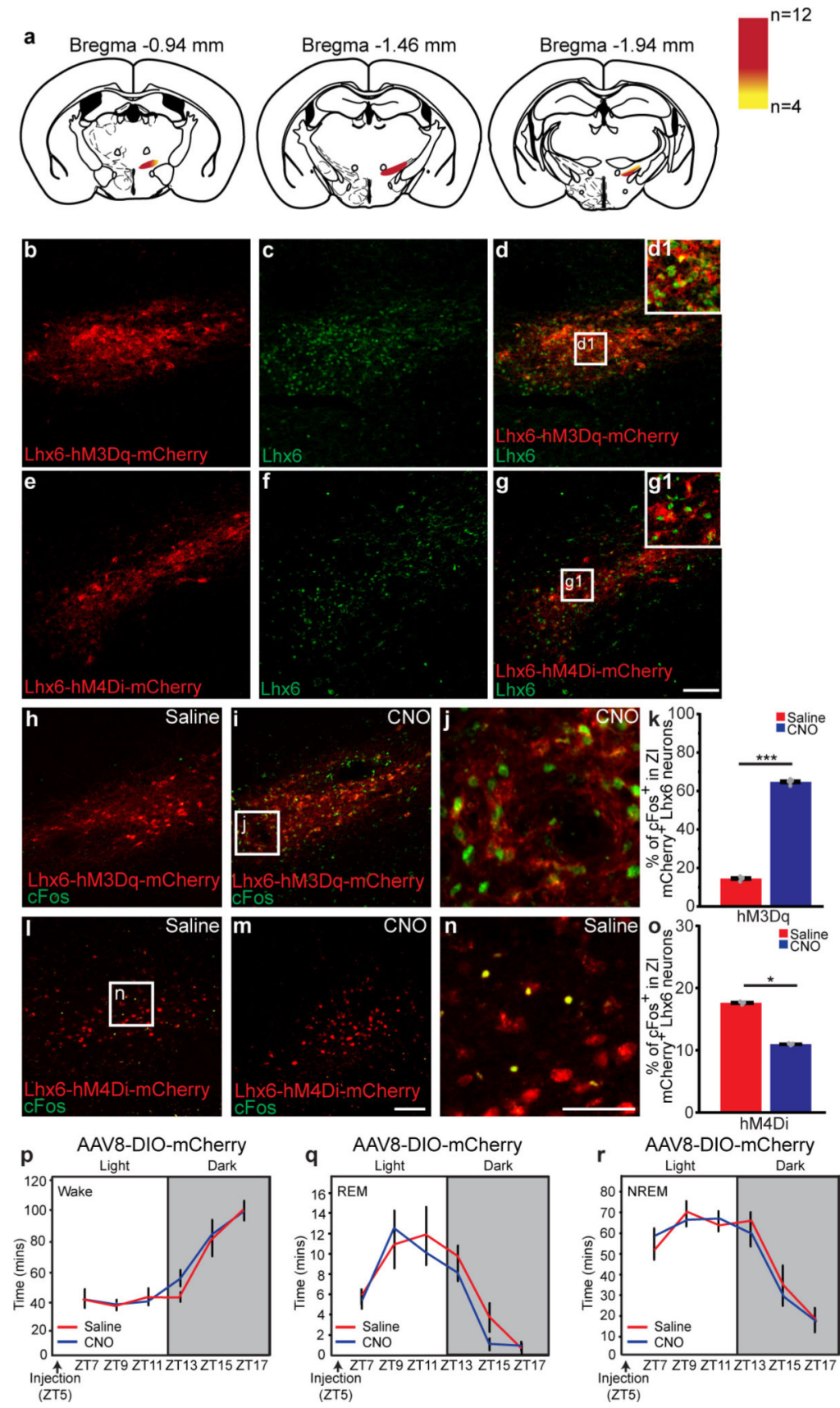

Extended Data Figure 7. Distribution of AAV-DREADD infection sites in the zona incerta of Lhx6-cre mice and CNO activates Fos expression in AAV-DIO-hM3Dq-injected Lhx6-Cre mice, whereas CNO does not affect sleep in AAV-DIO-mCherry-injected Lhx6-Cre mice

Continued from Fig. 4. a, Schematics showing distribution of DREADD-infected (hM3Dq or hM4Di mice) neurons (red) across the rostrocaudal extent of the zona incerta after AAVDREADD was injected into the zona incerta of Lhx6-cre mice. Infection is specific to the zona incerta in 12 Lhx6-cre mice (red colour indicates infection site from all 12 mice).

Yellow colour indicates additional infected areas seen in 4 out of 12 mice. b-d,

Representative images showing co-expression of hM3Dq DREADD in the zona incerta of Lhx6-cre mice (b, red) with Lhx6 immunostaining (c, green). The merged image is shown in 
$\mathbf{d}$, inset shows a magnified image of $\mathbf{d}$. $\mathbf{e}-\mathbf{g}$, Representative images showing co-expression of AAV-hM4Di infection in the zona incerta of Lhx6-cre mice (e, red) with Lhx6 immunostaining (f, green). A merged image is shown in $\mathbf{g}$; inset shows a magnified image of g. Scale bar, $100 \mu \mathrm{m}$. h-j, Representative images showing hM3Dq-mCherry expression (red) and Fos immunostaining in the zona incerta of Lhx6-cre mice after saline (h) or CNO (i) injection. A magnified image of $\mathbf{i}$ is shown in $\mathbf{j}$. $\mathbf{k}$, The percentage $\mathrm{Fos}^{+}$neurons in hM3Dq-mCherry ${ }^{+}$Lhx6-expressing neurons in the zona incerta in mice injected with saline or CNO. ${ }^{* * *} P<0.001$, two-tailed paired $t$-test; $n=4$ mice, 8 sections for saline; $n=5$ mice, 8 sections for CNO. l-n, Representative images showing hM4Di-mCherry expression (red) and Fos immunostaining (green) in the zona incerta of Lhx6-Cre mice after saline (l) or $\mathrm{CNO}(\mathbf{m})$ injection. A magnified image of $\mathbf{l}$ is shown in $\mathbf{n}$. Scale bars, $100 \mu \mathrm{m}$. o, The percentage $\mathrm{Fos}^{+}$neurons in hM4Di-mCherry ${ }^{+} \mathrm{Lhx6}$-expressing neurons in the zona incerta in mice injected with saline or CNO. $* P<0.05$, two-tailed paired $t$-test; $n=3$ mice, 8 sections for saline; $n=3$ mice, 8 sections for CNO. Data are mean \pm s.e.m. o-q, Saline- or CNO-injection at ZT5 in the AAV8-DIO-mCherry group shows that CNO injection does not affect sleep. EEG analysis showing amount of time spent across ZT in wake (o), REM sleep (p) and NREM sleep (q). $P>0.1$ for all behavioural readouts, two-way ANOVA followed by Sidak's post hoc test, $n=4$ mice for saline, $n=4$ mice for CNO. 
a

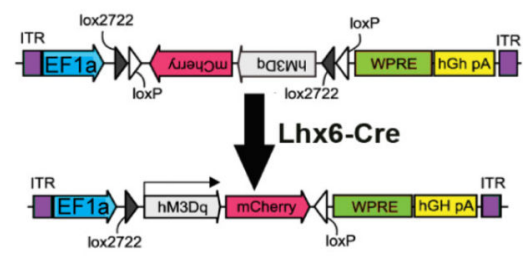

b

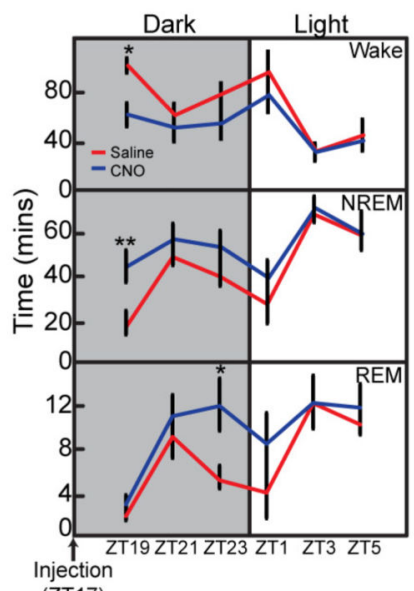

d

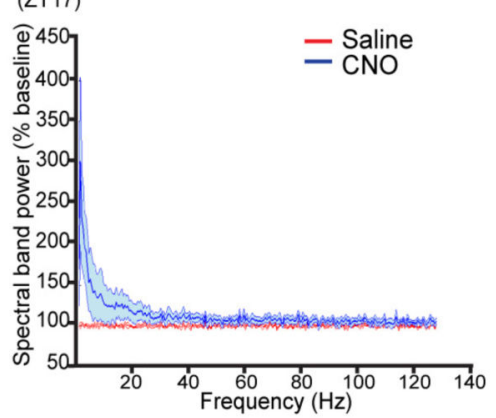

f

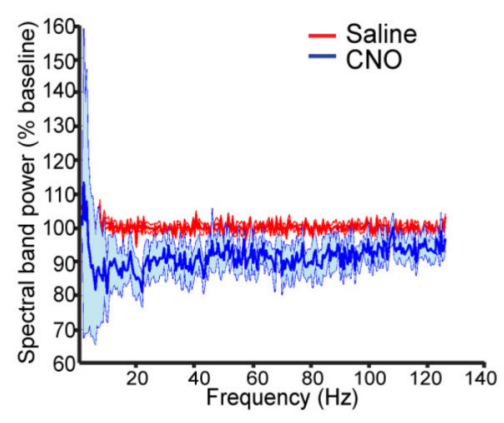

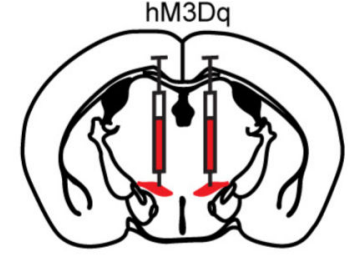

c
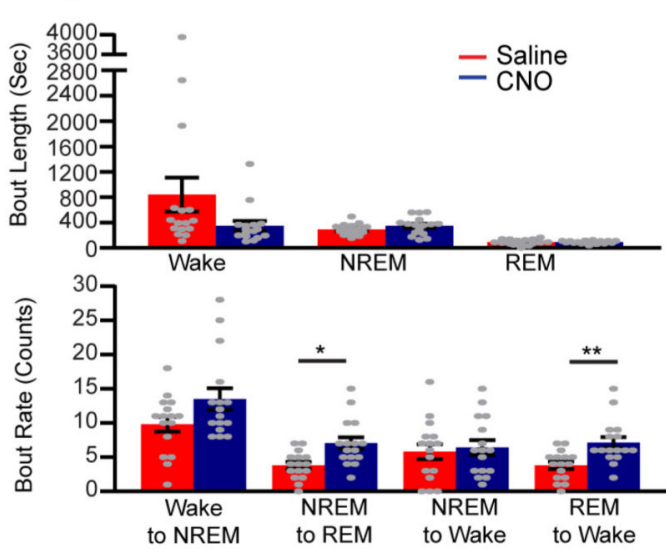

e
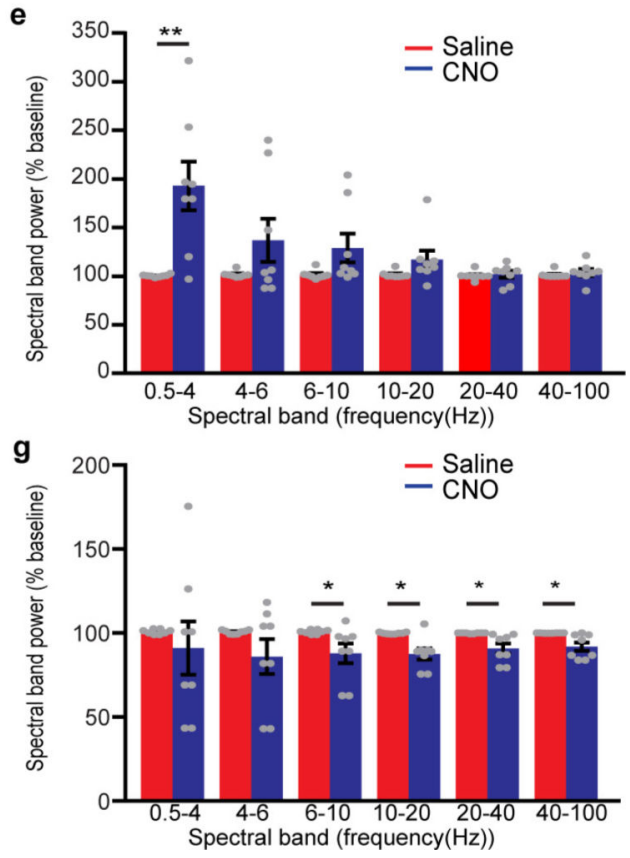

Extended Data Figure 8. Additional data from $G_{q}$ DREADD-injected Lhx6-cre mice Continued from Fig. 4. a, Schematics showing the construct of the AAV-DIO-hM3DqmCherry virus and the injection site of virus into the zona incerta of $L h x 6$-cre mice. b, EEG analysis showing the percentage of time in 2-h bins spent in wake (top), NREM sleep (middle) and REM sleep (bottom) after injection of saline or CNO at ZT17 in hM3Dq groups. c, Bout length quantification (top) showing durations for wake, NREM and REM episodes and bout count (bottom) showing sleep-wake transitions with saline or CNO injection in hM3Dq groups. d, FFT analysis of EEG power spectrum frequency of NREM stage of CNO-injected AAV-DIO-hM3Dq-infected mice (blue) compared to control (saline- 
injection, red). e, Grouped EEG frequency data obtained from spectrum band analysis of saline- (red) and CNO-injected (blue) hM3Dq mice. f, FFT analysis of EEG power spectrum frequency of the wake stage of CNO-injected AAV-DIO-hM3Dq-infected mice (blue) compared to control (saline-injection, red). g, Grouped EEG frequency data obtained from spectrum band analysis of saline- (red) and CNO-injected (blue) hM3Dq mice. Data are mean \pm s.e.m. $* P<0.05, * * P<0.01$; two-way ANOVA followed by Sidak's post hoc test $(\mathbf{b}$, $\mathbf{c}, \mathbf{e}, \mathbf{g}) . n=8$ mice $(\mathbf{b}-\mathbf{g})$.

a

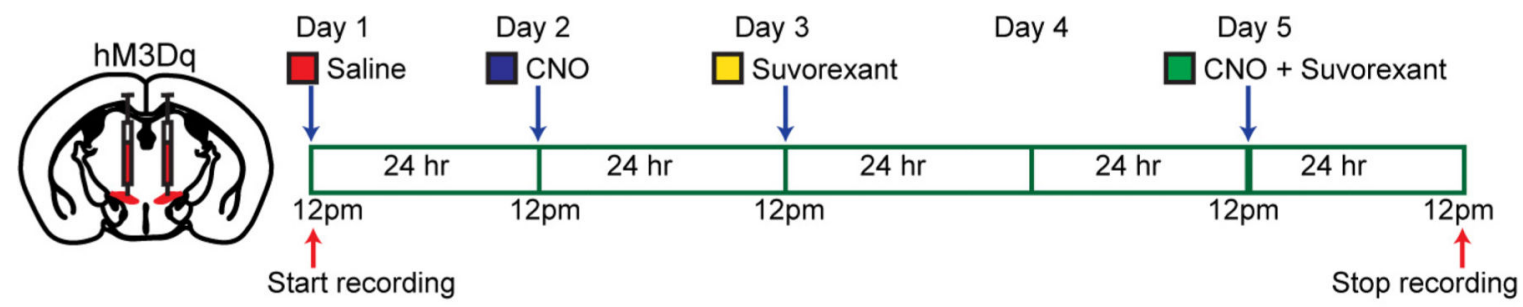

b
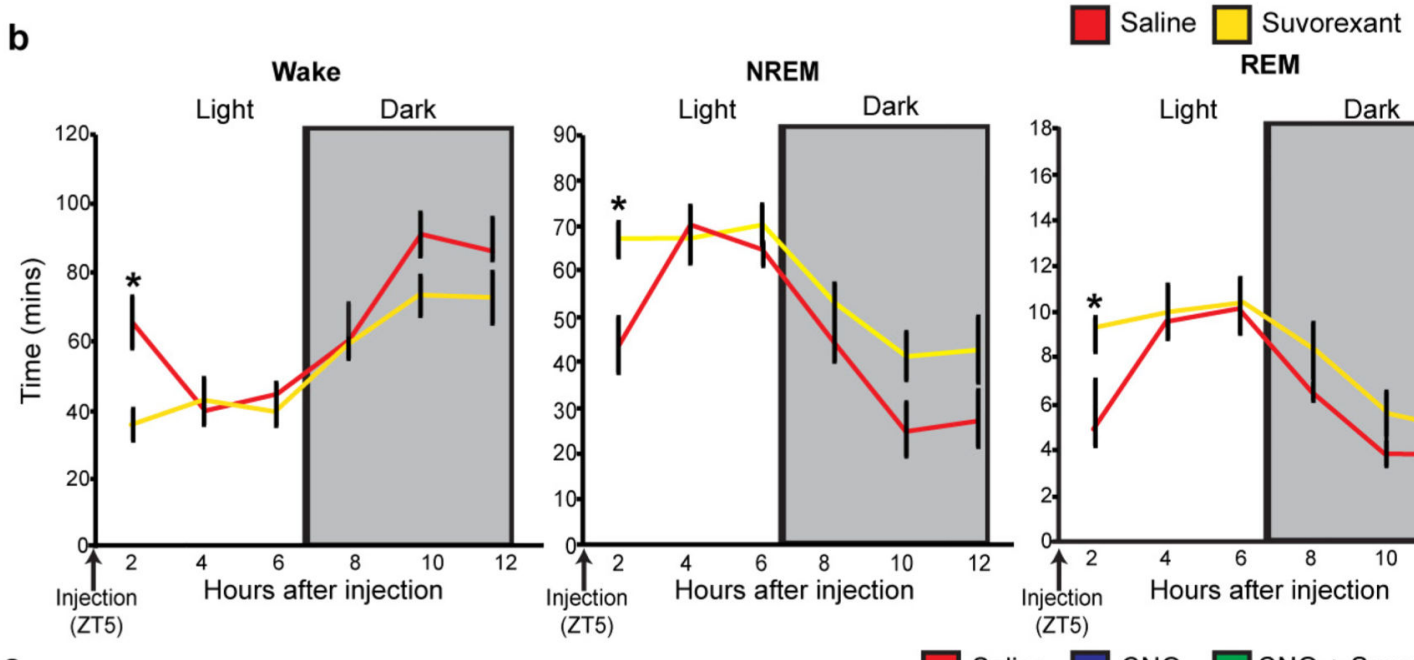

C
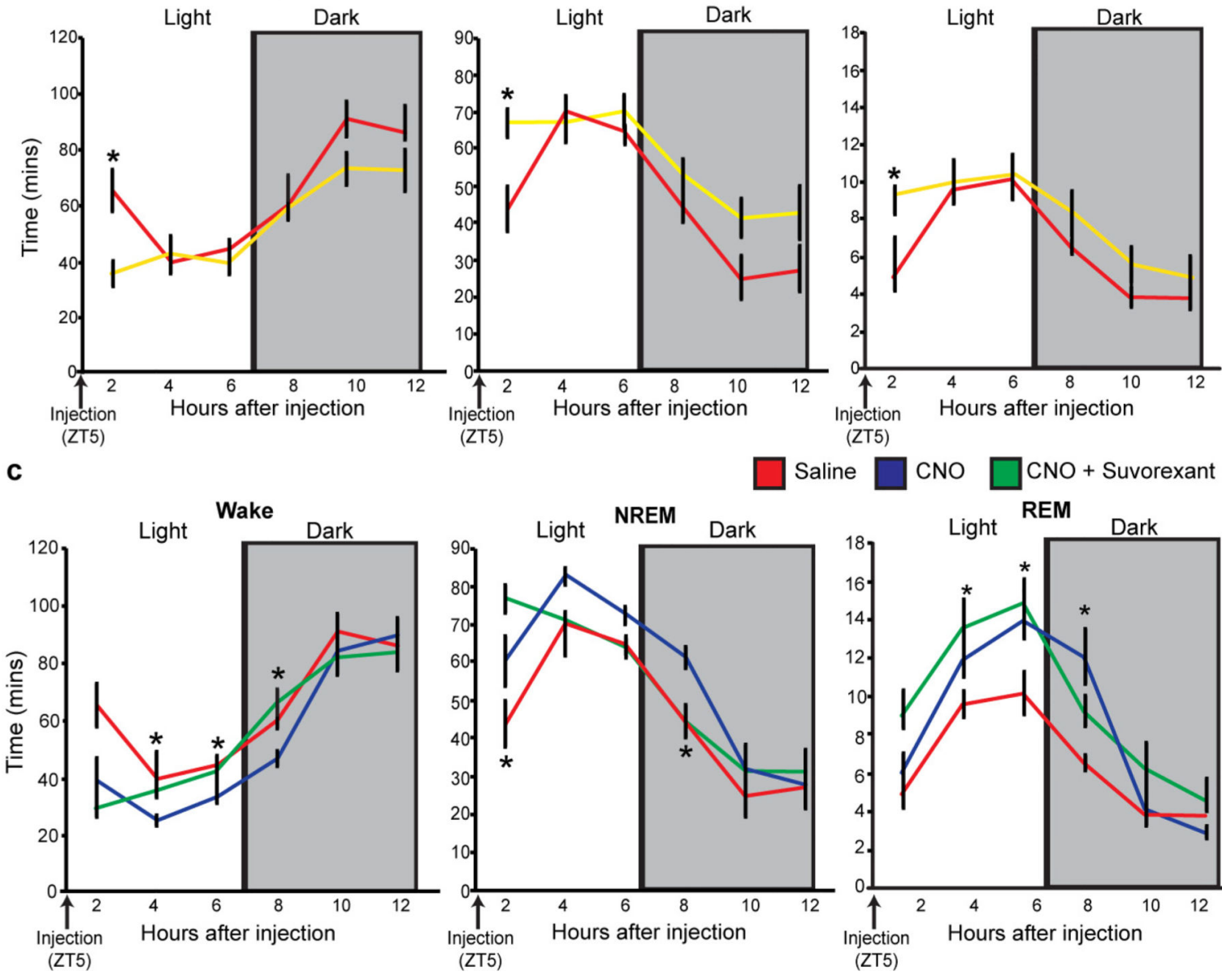

Extended Data Figure 9. The dual orexin receptor antagonist suvorexant can increase both NREM and REM sleep 
Continued from Fig. 4. a, Schematic showing sleep recording paradigm (EEG and EMG recording) for experiments with suvorexant. $\mathbf{b}$, EEG analysis showing the percentage of time in 2-h bins spent in wake (left), NREM sleep (middle) and REM sleep (right) after injection of saline or suvorexant at ZT5. c, EEG analysis showing the percentage of time in 2-h bins. The data show that suvorexant does not show a clear additive effect in combination with CNO in regulating time spent in wake (left), NREM sleep (middle) and REM sleep (right) when co-administered to mice expressing $\mathrm{G}_{\mathrm{q}}$ DREADDs in Lhx6 $6^{+}$neurons in the zona incerta in the first $2 \mathrm{~h}$ after administration. Data are mean \pm s.e.m. $* P<0.05$; two-way ANOVA followed by Sidak's post hoc test, $n=10$ mice. 
a
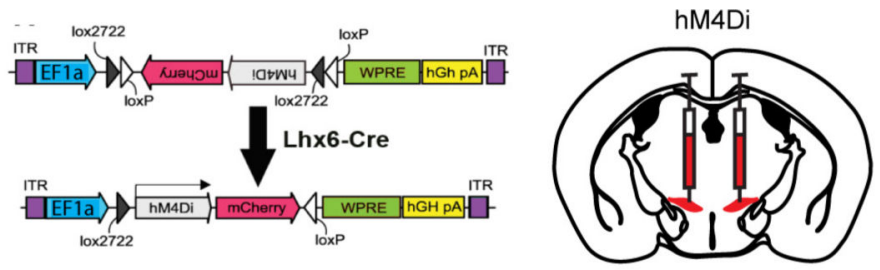

b
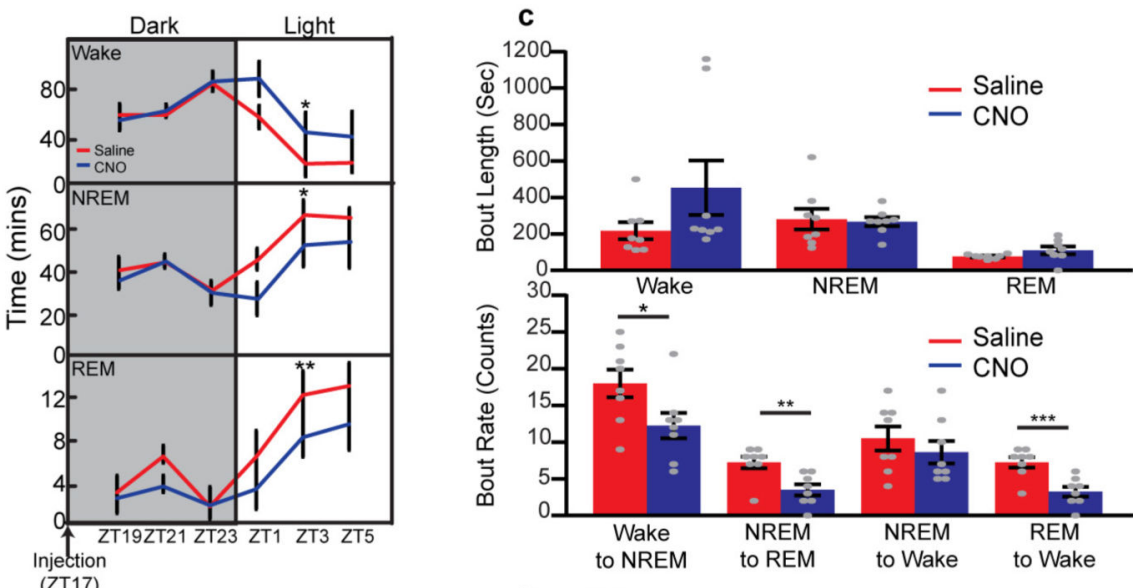

d
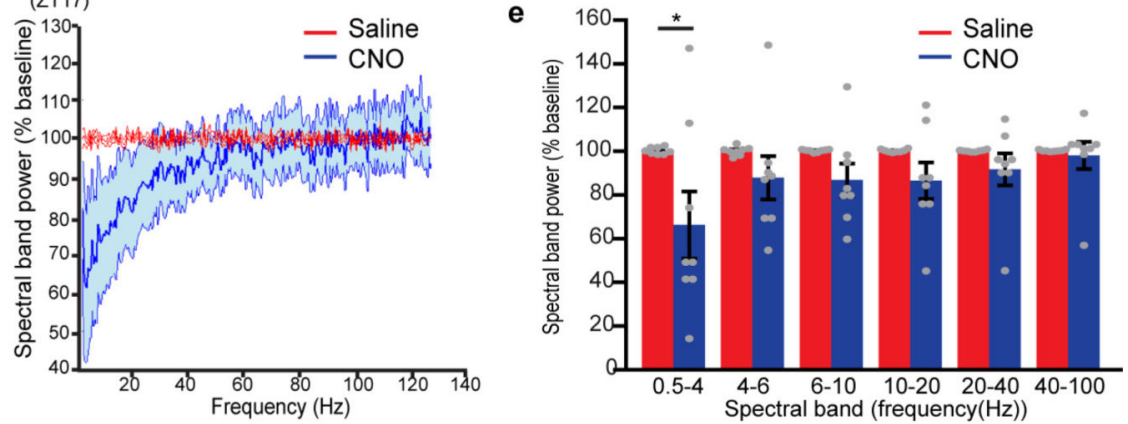

f
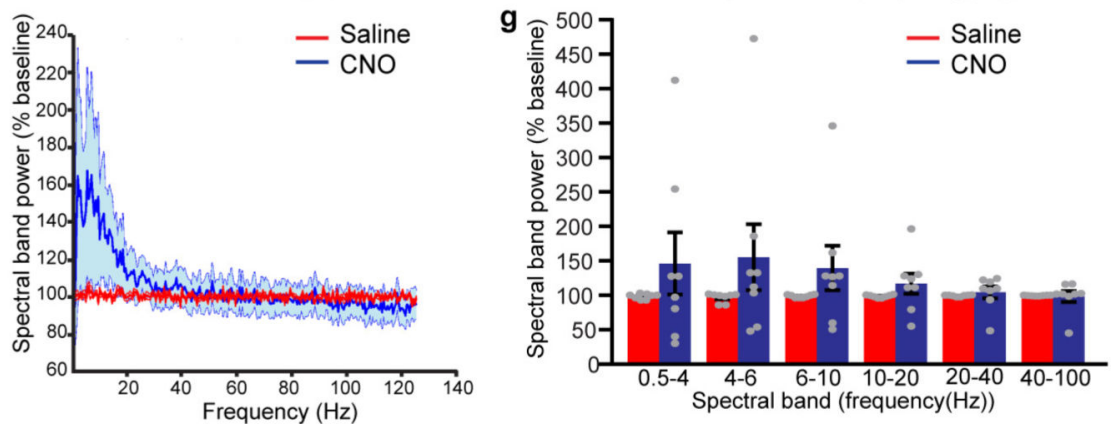

Extended Data Figure 10. Additional data from AAV-DIO-hM4Di-injected Lhx6-cre mice Continued from Fig. 4. a, Schematics showing the construct of AAV-DIO-hM4Di-mCherry virus and the injection sites of virus into the zona incerta of Lhx6-cre mice. b, EEG analysis showing the percentage of time in 2-h bins spent in wake (top), NREM sleep (middle) and REM sleep (bottom) after injection of saline or CNO at ZT17 in hM4Di groups. c, Bout length quantification (top) showing duration of wake, NREM and REM episodes and bout count (bottom) showing sleep-wake transitions with saline or CNO injection in hM4Di groups. d, FFT analysis of EEG power spectrum frequency of NREM stage of CNO-injected AAV-DIO-hM4Di (blue) compared to control (saline-injection, red). e, Grouped EEG 
frequency data of spectrum band analysis between saline- (red) and CNO-injected (blue) hM4Di groups. f, FFT analysis of EEG power spectrum frequency of NREM stage of CNOinjected AAV-DIO-hM4Di (blue) compared to control (saline-injection, red). g, Grouped EEG frequency data of spectrum band analysis between saline- (red) and CNO-injected (blue) hM4Di groups. Data are mean \pm s.e.m. $* P<0.05$, $* * P<0.01$, $* * * P<0.001$; two-way ANOVA followed by Sidak's post hoc test. $n=8$ mice.

\section{Acknowledgments}

We thank L. de Lecea and A. Gittis for providing mice; M. Pletnikov for help with behavioural analysis; and M. Wu, V. Mongrain, R. Kuruvila, D. Lee, J. Bedont and W. Yap for comments on the manuscript. This work was supported by a Johns Hopkins Discovery Fund award to S.B. and S.H. S.P.B. is supported by a Klingenstein-Simons Foundation Fellowship in the Neurosciences.

\section{References}

1. Weber F, Dan Y. Circuit-based interrogation of sleep control. Nature. 2016; 538:51-59. [PubMed: 27708309]

2. Chemelli RM, et al. Narcolepsy in orexin knockout mice: molecular genetics of sleep regulation. Cell. 1999; 98:437-451. [PubMed: 10481909]

3. Saper CB, Fuller PM, Pedersen NP, Lu J, Scammell TE. Sleep state switching. Neuron. 2010; 68:1023-1042. [PubMed: 21172606]

4. Saito YC, et al. GABAergic neurons in the preoptic area send direct inhibitory projections to orexin neurons. Front. Neural Circuits. 2013; 7:192. [PubMed: 24348342]

5. Mitrofanis J. Some certainty for the "zone of uncertainty"? Exploring the function of the zona incerta. Neuroscience. 2005; 130:1-15. [PubMed: 15561420]

6 . Liu J, et al. Frequency-selective control of cortical and subcortical networks by central thalamus. eLife. 2015; 4:e09215. [PubMed: 26652162]

7. Jurkowlaniec E, Trojniar W, Tokarski J. The EEG activity after lesions of the diencephalic part of the zona incerta in rats. Acta Physiol. Pol. 1990; 41:85-97. [PubMed: 2136322]

8. Shimogori T, et al. A genomic atlas of mouse hypothalamic development. Nat. Neurosci. 2010; 13:767-775. [PubMed: 20436479]

9. Gong S, et al. A gene expression atlas of the central nervous system based on bacterial artificial chromosomes. Nature. 2003; 425:917-925. [PubMed: 14586460]

10. Fogarty M, et al. Spatial genetic patterning of the embryonic neuroepithelium generates GABAergic interneuron diversity in the adult cortex. J. Neurosci. 2007; 27:10935-10946. [PubMed: 17928435]

11. Liodis P, et al. Lhx 6 activity is required for the normal migration and specification of cortical interneuron subtypes. J. Neurosci. 2007; 27:3078-3089. [PubMed: 17376969]

12. Taniguchi $\mathrm{H}$, et al. A resource of Cre driver lines for genetic targeting of GABAergic neurons in cerebral cortex. Neuron. 2011; 71:995-1013. [PubMed: 21943598]

13. Oh SW, et al. A mesoscale connectome of the mouse brain. Nature. 2014; 508:207-214. [PubMed: 24695228]

14. Wickersham IR, et al. Monosynaptic restriction of transsynaptic tracing from single, genetically targeted neurons. Neuron. 2007; 53:639-647. [PubMed: 17329205]

15. Venner A, Anaclet C, Broadhurst RY, Saper CB, Fuller PM. A novel population of wake-promoting GABAergic neurons in the ventral lateral hypothalamus. Curr. Biol. 2016; 26:2137-2143. [PubMed: 27426511]

16. Veasey SC, Yeou-Jey H, Thayer P, Fenik P. Murine multiple sleep latency test: phenotyping sleep propensity in mice. Sleep. 2004; 27:388-393. [PubMed: 15164889]

17. Li Y, Gao XB, Sakurai T, van den Pol AN. Hypocretin/orexin excites hypocretin neurons via a local glutamate neuron-A potential mechanism for orchestrating the hypothalamic arousal system. Neuron. 2002; 36:1169-1181. [PubMed: 12495630] 
18. Petreanu L, Mao T, Sternson SM, Svoboda K. The subcellular organization of neocortical excitatory connections. Nature. 2009; 457:1142-1145. [PubMed: 19151697]

19. Karnani MM, Szabó G, Erdélyi F, Burdakov D. Lateral hypothalamic GAD65 neurons are spontaneously firing and distinct from orexin- and melanin-concentrating hormone neurons. J. Physiol. 2013; 591:933-953. [PubMed: 23184514]

20. Hu H, Ma Y, Agmon A. Submillisecond firing synchrony between different subtypes of cortical interneurons connected chemically but not electrically. J. Neurosci. 2011; 31:3351-3361. [PubMed: 21368047]

21. Skeldon AC, Dijk DJ, Derks G. Mathematical models for sleep-wake dynamics: comparison of the two-process model and a mutual inhibition neuronal model. PLoS ONE. 2014; 9:e103877. [PubMed: 25084361]

22. Salvatierra J, et al. The LIM homeodomain factor Lhx 2 is required for hypothalamic tanycyte specification and differentiation. J. Neurosci. 2014; 34:16809-16820. [PubMed: 25505333]

23. Armbruster BN, Li X, Pausch MH, Herlitze S, Roth BL. Evolving the lock to fit the key to create a family of G protein-coupled receptors potently activated by an inert ligand. Proc. Natl Acad. Sci. USA. 2007; 104:5163-5168. [PubMed: 17360345]

24. Hoyer D, et al. Distinct effects of IPSU and suvorexant on mouse sleep architecture. Front. Neurosci. 2013; 7:235. [PubMed: 24368893]

25. Chung $\mathrm{S}$, et al. Identification of preoptic sleep neurons using retrograde labelling and gene profiling. Nature. 2017; 545:477-481. [PubMed: 28514446]

26. Anaclet $\mathrm{C}$, et al. The GABAergic parafacial zone is a medullary slow wave sleep-promoting center. Nat. Neurosci. 2014; 17:1217-1224. [PubMed: 25129078]

27. Sherin JE, Shiromani PJ, McCarley RW, Saper CB. Activation of ventrolateral preoptic neurons during sleep. Science. 1996; 271:216-219. [PubMed: 8539624]

28. Urbain N, Deschênes M. Motor cortex gates vibrissal responses in a thalamocortical projection pathway. Neuron. 2007; 56:714-725. [PubMed: 18031687]

29. Humphreys BD, et al. Fate tracing reveals the pericyte and not epithelial origin of myofibroblasts in kidney fibrosis. Am. J. Pathol. 2010; 176:85-97. [PubMed: 20008127]

30. Peron SP, Freeman J, Iyer V, Guo C, Svoboda K. A cellular resolution map of barrel cortex activity during tactile behavior. Neuron. 2015; 86:783-799. [PubMed: 25913859]

31. Rodríguez CI, et al. High-efficiency deleter mice show that FLPe is an alternative to Cre-loxP. Nat. Genet. 2000; 25:139-140. [PubMed: 10835623]

32. Thompson CL, et al. Molecular and anatomical signatures of sleep deprivation in the mouse brain. Front. Neurosci. 2010; 4:165. [PubMed: 21088695]

33. Lee JH, et al. Global and local fMRI signals driven by neurons defined optogenetically by type and wiring. Nature. 2010; 465:788-792. [PubMed: 20473285]

34. Paxinos, GFK. The Mouse Brain in Sterotaxic Coordinates. 4. Academic; 2012.

35. Cox CD, et al. Discovery of the dual orexin receptor antagonist [(7R)-4-(5-chloro-1,3benzoxazol-2-yl)-7-methyl-1,4-diazepan-1-yl][5-methyl-2-(2H-1,2,3-triazol-2yl)phenyl]methanone (MK-4305) for the treatment of insomnia. J. Med. Chem. 2010; 53:53205332. [PubMed: 20565075]

36. Wickersham IR, Sullivan HA. Rabies viral vectors for monosynaptic tracing and targeted transgene expression in neurons. Cold Spring Harb. Protoc. 2015; 2015:375-385. [PubMed: 25834254]

37. Wickersham IR, Sullivan HA, Seung HS. Production of glycoprotein-deleted rabies viruses for monosynaptic tracing and high-level gene expression in neurons. Nat. Protocols. 2010; 5:595-606. [PubMed: 20203674]

38. Kohara K, et al. Cell type-specific genetic and optogenetic tools reveal hippocampal CA2 circuits. Nat. Neurosci. 2014; 17:269-279. [PubMed: 24336151]

39. Miyamichi K, et al. Cortical representations of olfactory input by trans-synaptic tracing. Nature. 2011; 472:191-196. [PubMed: 21179085]

40. Subach FV, Piatkevich KD, Verkhusha VV. Directed molecular evolution to design advanced red fluorescent proteins. Nat. Methods. 2011; 8:1019-1026. [PubMed: 22127219] 
41. Kim J, Matney CJ, Blankenship A, Hestrin S, Brown SP. Layer 6 corticothalamic neurons activate a cortical output layer, layer 5a. J. Neurosci. 2014; 34:9656-9664. [PubMed: 25031405]

42. Blackshaw S, et al. Genomic analysis of mouse retinal development. PLoS Biol. 2004; 2:E247. [PubMed: 15226823] 

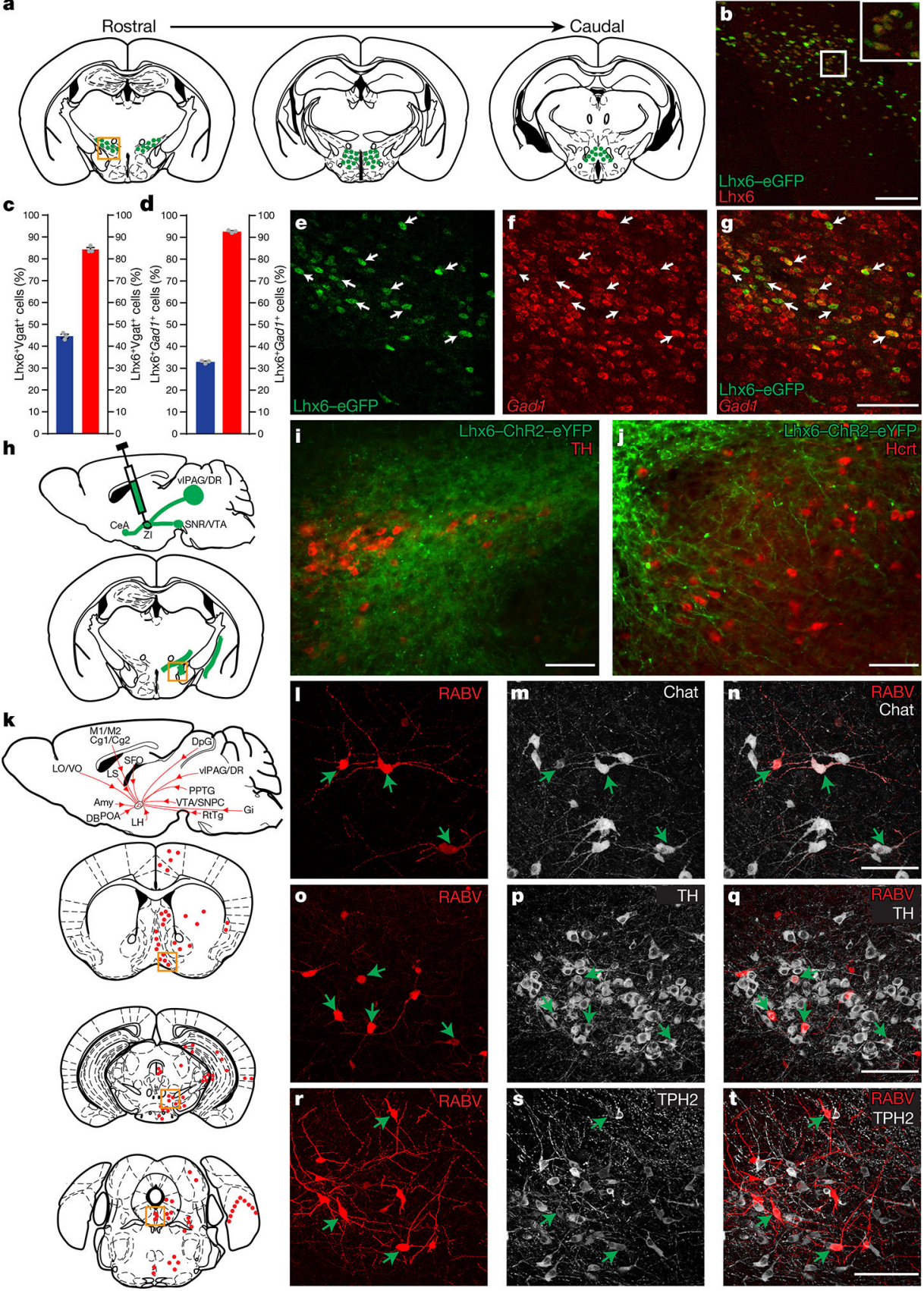

Figure 1. Lhx6 ${ }^{+}$neurons in the VZI are GABAergic, receive synaptic inputs from sleepregulating regions, and project to $\mathrm{Hcrt}^{+}$neurons in the lateral hypothalamus

a, Distribution of $\operatorname{Lhx}^{+}$neurons in the hypothalamus. Box indicates the location of $\operatorname{Lhx} 6^{+}$ neurons (green) in the VZI in b. b, Co-expression of eGFP (green) in the Lhx6-eGFP line with Lhx6 (red) in the VZI. Inset, magnification of the boxed area. c, The percentage of Lhx $6^{+}$tdTomato $^{+}$cells (blue) in the VZI relative to all tdTomato ${ }^{+}$cells in the VZI in Slc32a1-cre; $A i 9$ mice. The percentage of $\mathrm{Lhx}^{+} \mathrm{tdTomato}^{+}$neurons (red) relative to all Lhx6 $6^{+}$neurons in the VZI in Slc32a1-cre;Ai9 mice. $n=3$ mice, eight sections per group. d, The percentage of $\mathrm{Lhx}^{+} \mathrm{Gadl}^{+}$cells (blue) relative to all $\mathrm{Gadl}^{+}$neurons in the VZI. The 
percentage of $\mathrm{Lhx}^{+} \mathrm{Gadl}^{+}$cells (red) relative to all Lhx6 $6^{+}$cells in the VZI. $n=3$ mice, six sections per group. Data are mean \pm s.e.m. (c, d). e-g, Co-localization of Lhx6-eGFP (e, green) with Gad1 mRNA (f, red) in the VZI. Merged image is shown in g. h, ChR2-eYFP virus injection site in the zona incerta of the Lhx6-cre line. i, j, Distribution of ChR2-eYFPinfected Lhx6 ${ }^{+}$neurons (green) in the zona incerta (i) and projections of Lhx6 $6^{+}$neurons in the lateral hypothalamus (j). Tyrosine hydroxylase (TH) $(\mathbf{i}$, red), and Hcrt $(\mathbf{j}$, red) are shown. $\mathbf{k}$, Rabies virus (RABV) injection site in the zona incerta of $L h \times 6$-cre, and the distribution of neurons presynaptic to Lhx6 $6^{+}$VZI cells. $\mathbf{l}-\mathbf{n}, \mathrm{RABV}^{+}$cells in the nucleus of the diagonal band $\left(\mathbf{l}\right.$, red) stained with anti-Chat $\left(\mathbf{m}\right.$, grey). Merged image is shown in $\mathbf{n} . \mathbf{o}-\mathbf{q}, \mathrm{RABV}^{+}$ cells in the ventral tegmental area (o, red) stained with anti-tyrosine hydroxylase (p, grey). Merged image is shown in q. $\mathbf{r}-\mathbf{t}, \mathrm{RABV}^{+}$cells in the dorsal raphe nucleus (r, red) stained with anti-TPH2 (s, grey). Merged image is shown in t. Scale bars, $100 \mu \mathrm{m}$. Arrows show cells co-expressing relevant markers. Amy, amygdala; $\mathrm{CeA}$, central nucleus of the amygdala; $\mathrm{Cg} 1 / \mathrm{Cg} 2$, cingulate cortex; DB, diagonal band; DpG, motor-related nuclei of superior colliculus; Gi, midline gigantocellular nucleus of the medulla; LH, lateral hypothalamus; $\mathrm{LO} / \mathrm{VO}$, lateral/ventral orbital cortex; LS, lateral septum; M1/M2, frontal/secondary motor cortex; POA, preoptic area; PPTG, pedunculopontine tegmental nucleus; RtTg, midbrain reticular formation; SFO, subfornical organ; SNR/VTA, substantia nigra reticular part/ ventral tegmental area; vlPAG/DR, ventrolateral periaqueductal grey/dorsal raphe nucleus; VTA/SNPC, ventral tegmental area/substantia nigra pars compact; ZI, zona incerta. 

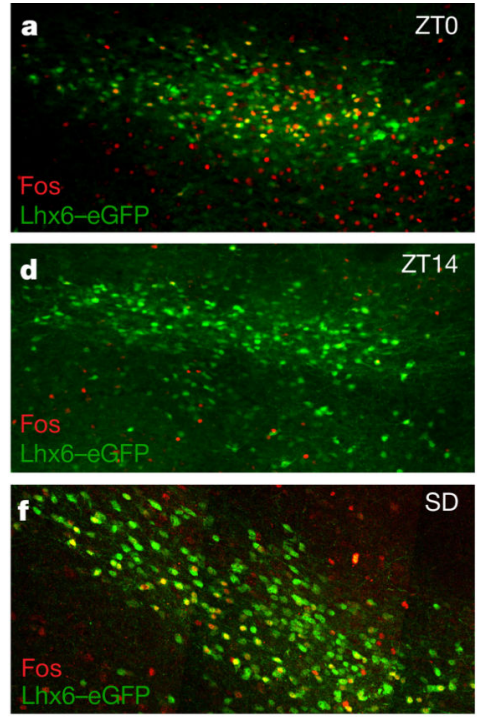

$\mathbf{i}$

$-70 \mathrm{mV}$
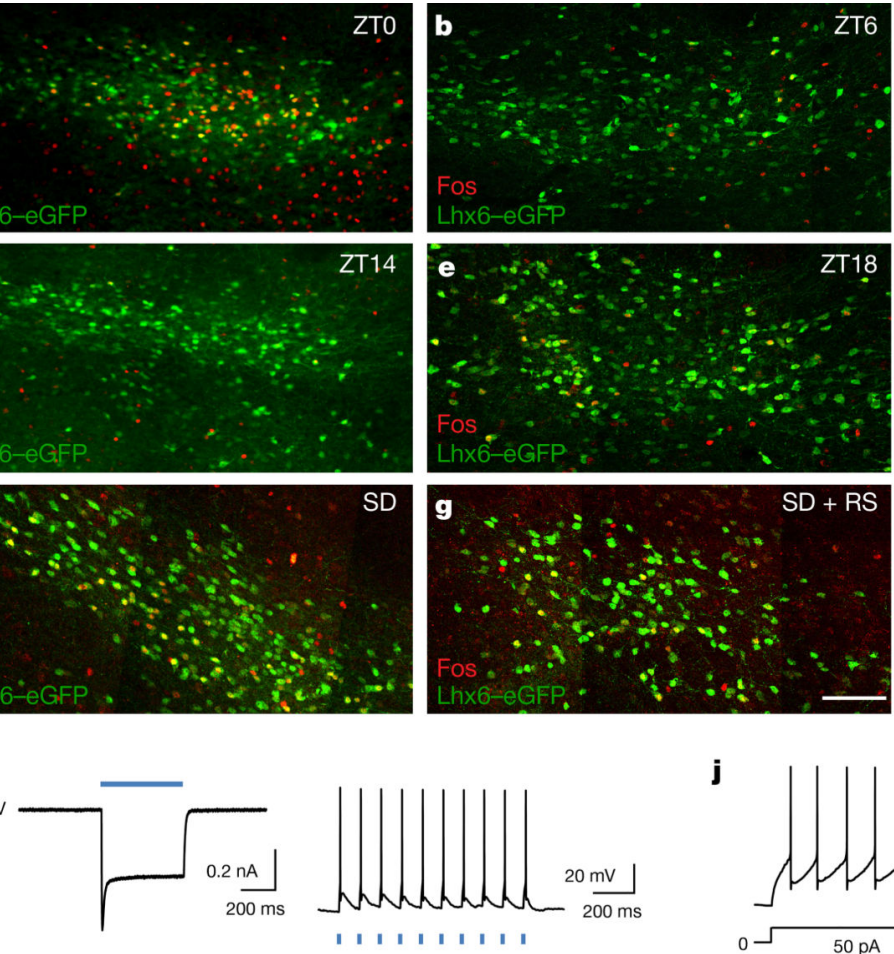

j

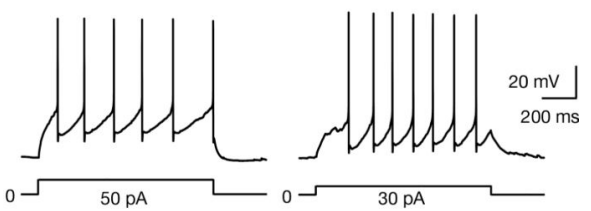

k Control $\quad \mathrm{CPP}+\mathrm{NBQX} \quad \mathrm{CPP}+\mathrm{NBQX}+\mathrm{CPP}+\mathrm{NBQX}$

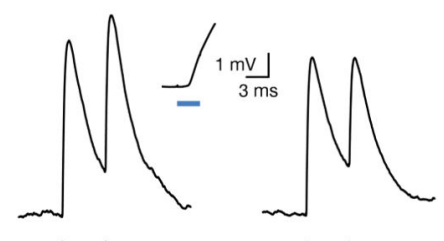
gabazine

m

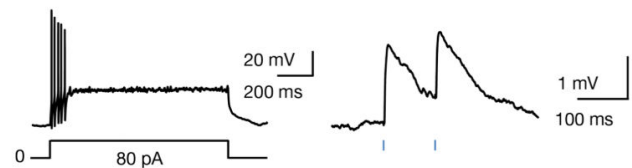

○
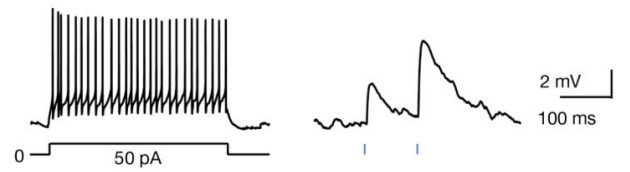

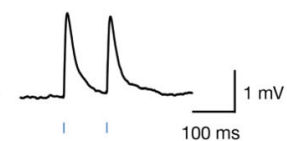

n

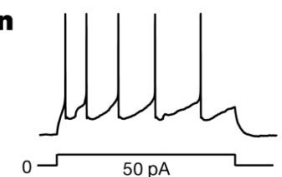

$\mathbf{p}$

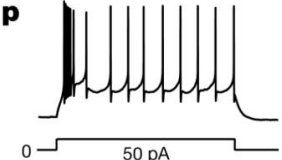

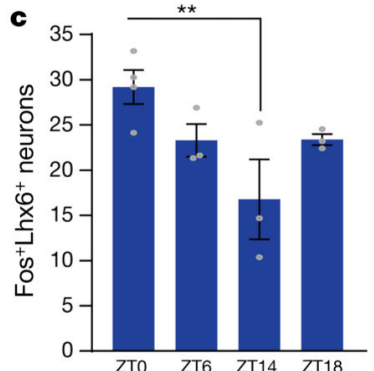

h

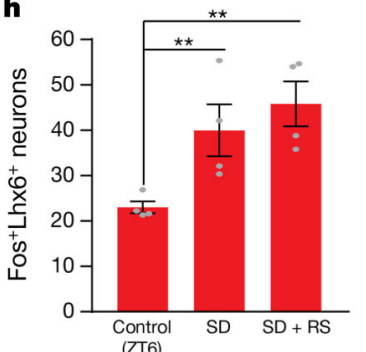

Figure 2. Lhx6 ${ }^{+}$VZI neurons are activated by sleep pressure and are presynaptic to $\mathrm{Hcrt}^{+}$and GABAergic neurons in the lateral hypothalamus

a, b, d-g, Representative images of Fos immunostaining (red) and Lhx6-eGFP expression (green) in VZI for ZT0 (a), ZT6 (b), ZT14 (d), ZT18 (e), following sleep deprivation (SD) (f) and sleep deprivation paired with recovery sleep (RS) (g). Scale bar, $100 \mu \mathrm{m}$. c, h, The percentage of $\mathrm{Fos}^{+} \mathrm{Lhx}^{+}$neurons in the zona incerta across ZT (c) and under sleep pressure (h). ${ }^{* *} P<0.01$; linear mixed-effect model fit by REML (h), two-tailed one-way ANOVA (c). $n=4$ mice per group, four brain sections analysed bilaterally per mouse. i, Average response to photostimulation $\left(500 \mathrm{~ms}\right.$ ) of a ChR2-expressing Lhx6 ${ }^{+}$VZI neuron (left; 
voltage-clamp) and action potentials elicited by photostimulation $(3 \mathrm{~ms}, 10 \mathrm{~Hz})$ from the same neuron (right; current-clamp). j, Responses of two $\mathrm{Hcrt}^{+}$neurons to depolarizing current steps. k, Postsynaptic responses from a $\mathrm{Hcrt}^{+}$neuron following photostimulation of $\mathrm{ChR} 2^{+} \mathrm{Lhx}^{+}{ }^{+}$neurons under control conditions (left), with glutamate receptor blockers NBQX (2,3-dioxo-6-nitro-1,2,3,4-tetrahydrobenzo[f]quinoxaline-7-sulfonamide disodium salt) and CPP ((RS)-3-(2-carboxypiperazin-4-yl)-propyl-1-phosphonic acid) (CPP + NBQX; middle), $\mathrm{CPP}, \mathrm{NBQX}$ and the $\mathrm{GABA}_{\mathrm{A}}$ receptor antagonist gabazine (6-imino-3-(4methoxyphenyl)-1(6H)-pyridazinebutanoic acid hydrobromide) (CPP + NBQX + gabazine; right) and after gabazine washout (far-right). Inset shows the onset of the postsynaptic response shown at an expanded timescale for the control. l, Amplitudes of the first postsynaptic response to photostimulation in control conditions, with NBQX and CPP, with NBQX, CPP and gabazine, and after gabazine washout. Two-way ANOVA with Bonferroni correction; control versus $\mathrm{CPP}+\mathrm{NBQX}, P=1$; $\mathrm{CPP}+\mathrm{NBQX}$ versus $\mathrm{CPP}+\mathrm{NBQX}+$ gabazine, $* * * P=0.00024$; control versus $\mathrm{CPP}+\mathrm{NBQX}+$ gabazine, $* * * P=0.00077$; $\mathrm{CPP}+$ NBQX versus CPP + NBQX + gabazine washout, $* P=0.0478 ; n=8$ Hcrt neurons from four mice. $\mathbf{m}-\mathbf{p}$, Responses to depolarizing current steps (left) and average short-latency postsynaptic potentials (right) in $\mathrm{Gad}^{+}$neurons following photostimulation of $\mathrm{ChR} 2^{+} \mathrm{Lhx} 6^{+}$ neurons. Data are mean \pm s.e.m. $(\mathbf{c}, \mathbf{h}, \mathbf{l})$. 
a

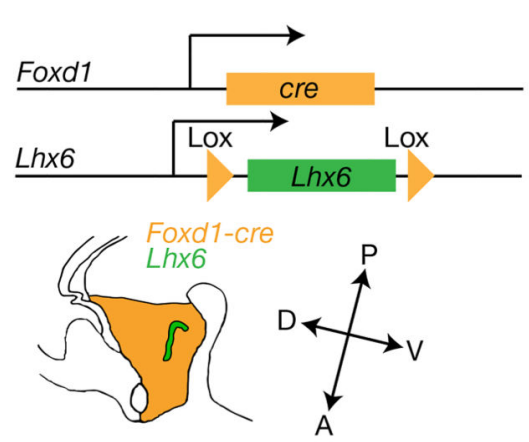

\section{C}

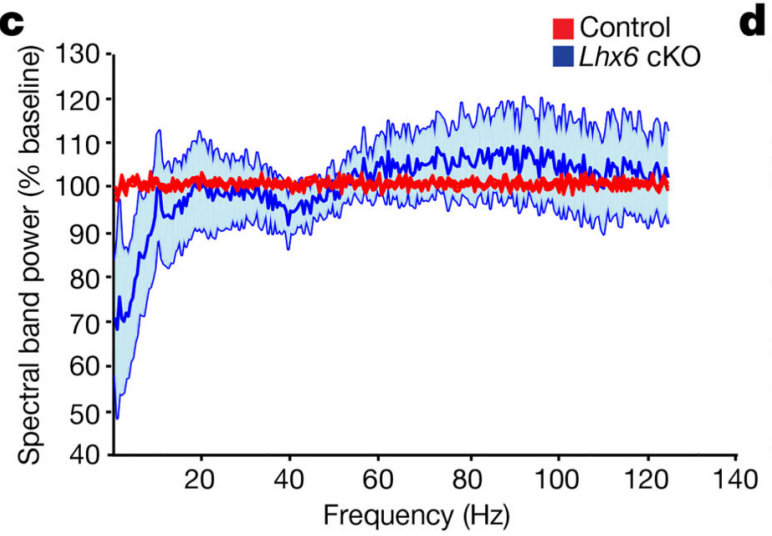

e

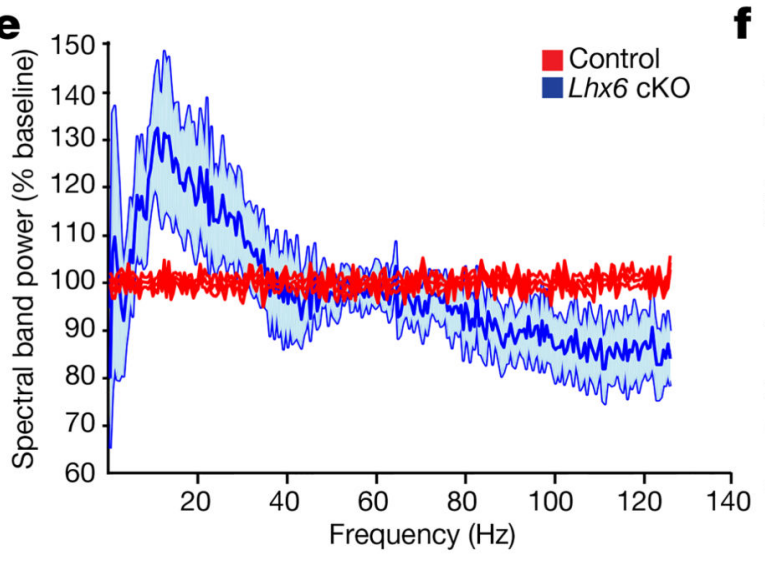

f b
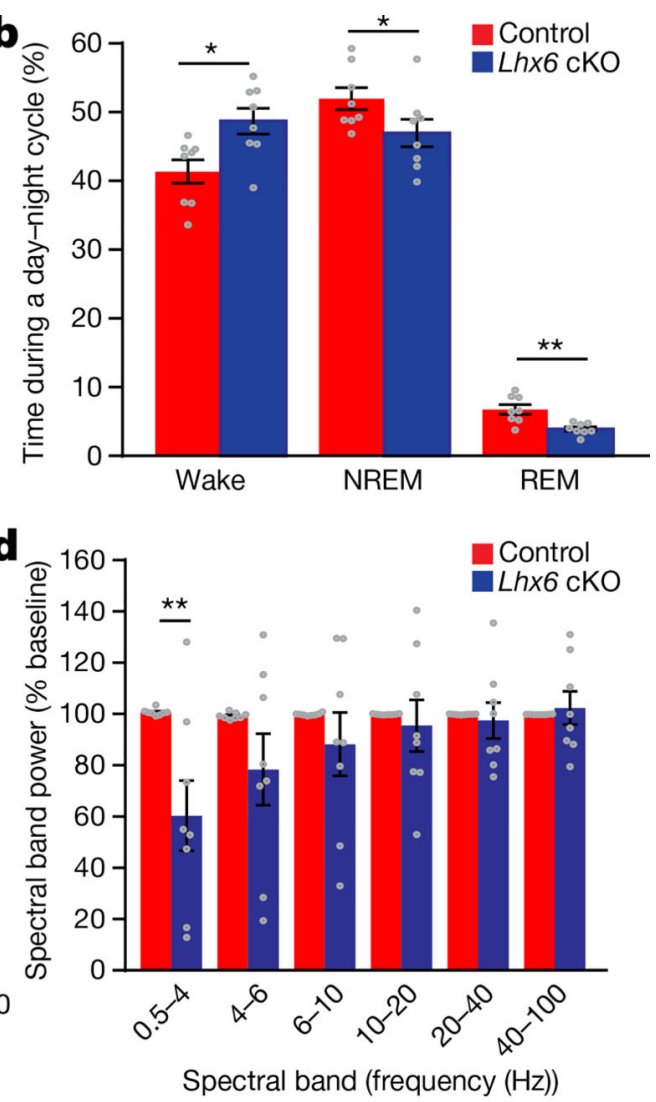

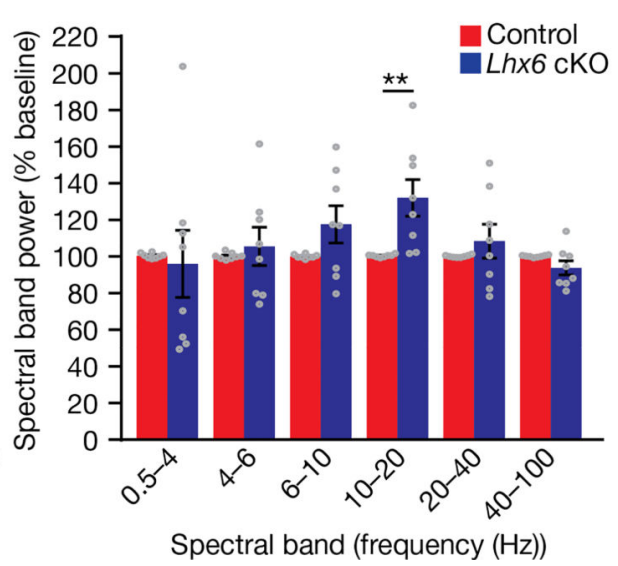

Figure 3. Selective loss of Lhx6 expression in the diencephalon reduces both NREM and REM sleep

a, Generation of diencephalic-specific Lhx6 conditional knockout (cKO) line by crossing Foxd1-cre and Lhx $\sigma^{\text {lox } / l o x}$ lines. A, anterior, D, dorsal, P, posterior, V, ventral. b, EEG/EMG analysis showing the percentage of the day-night cycle spent awake and in NREM and REM sleep in control (Foxd1-cre;Lhx $6^{\text {lox/+ }}$ ) and cKO groups. c, Fast Fourier transform (FFT) analysis of EEG power spectrum during NREM sleep of cKO (blue) compared to control (red) mice. d, The collapsed EEG frequency data are shown for control (red) and cKO (blue) mice. e, FFT analysis of EEG power spectrum frequency in cKO mice (blue) compared to 
controls (red). f, The collapsed EEG frequency data are shown for control (red) and cKO (blue) mice. $n=8$ control and $8 \mathrm{cKO}$ mice for all experiments. Data are mean \pm s.e.m. $* P<$ $0.05, * * P<0.01$; two-way ANOVA followed by Sidak's post hoc test. 
a
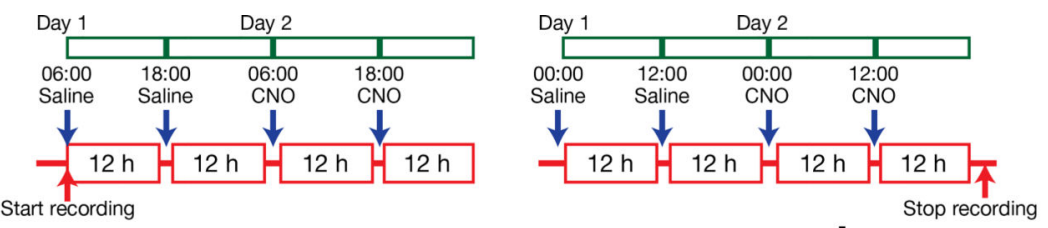

b
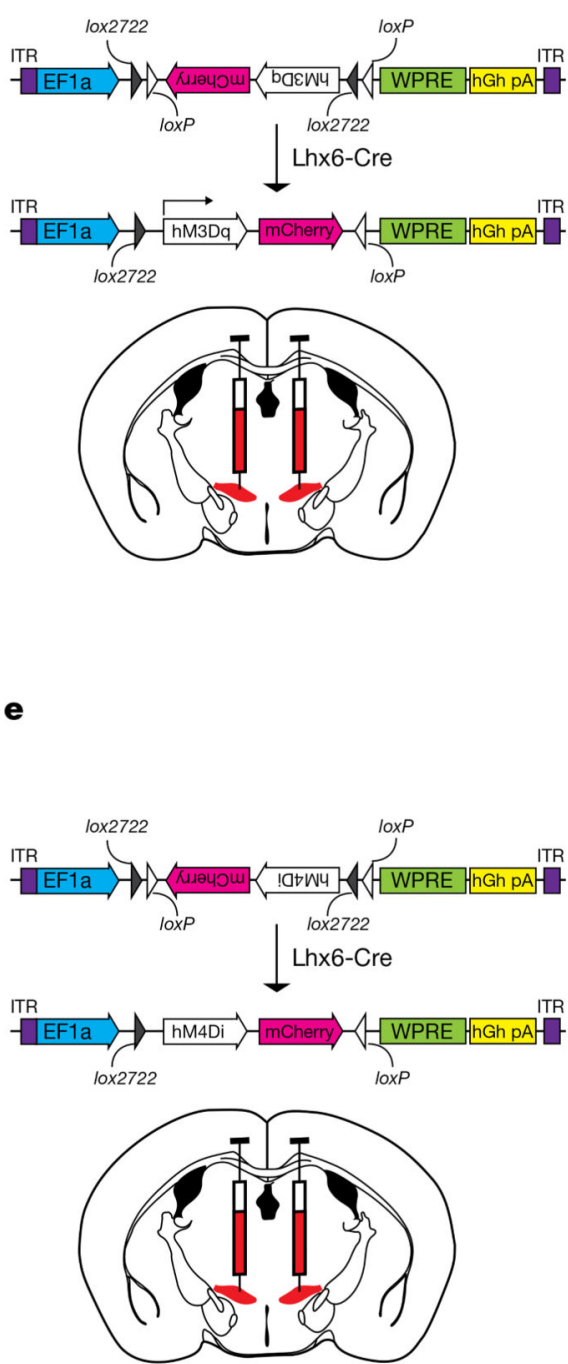

c
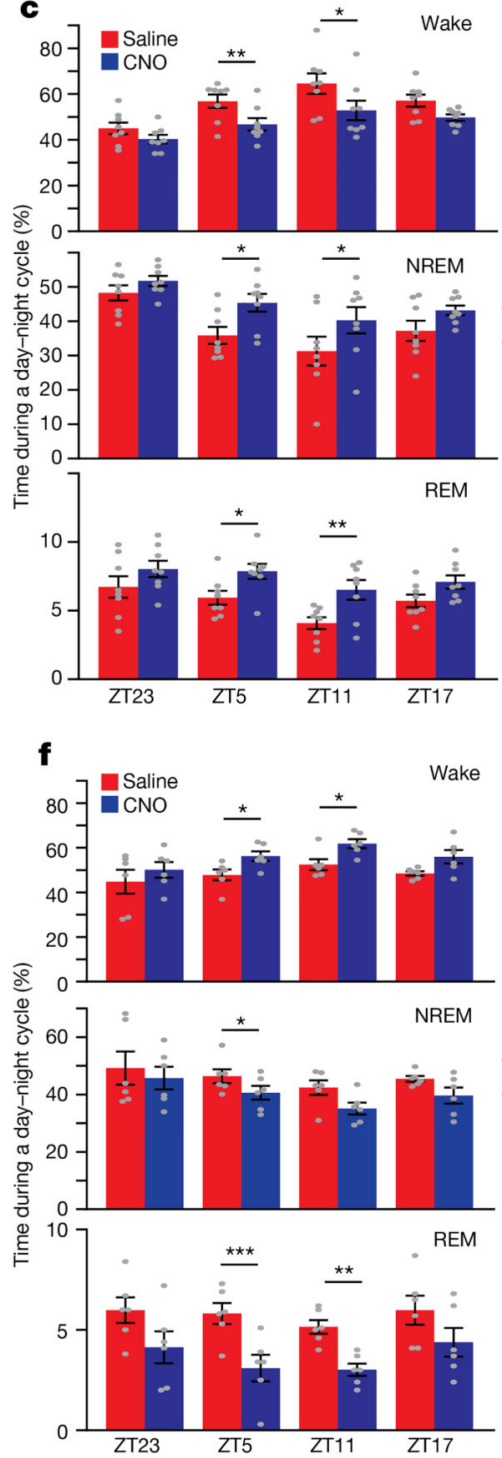

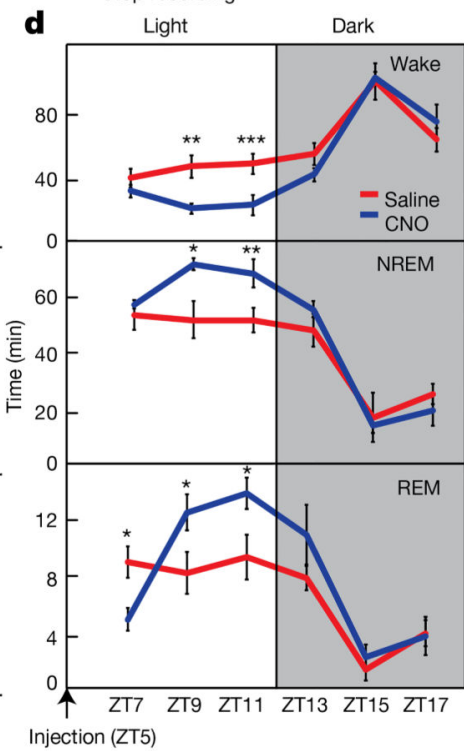

g

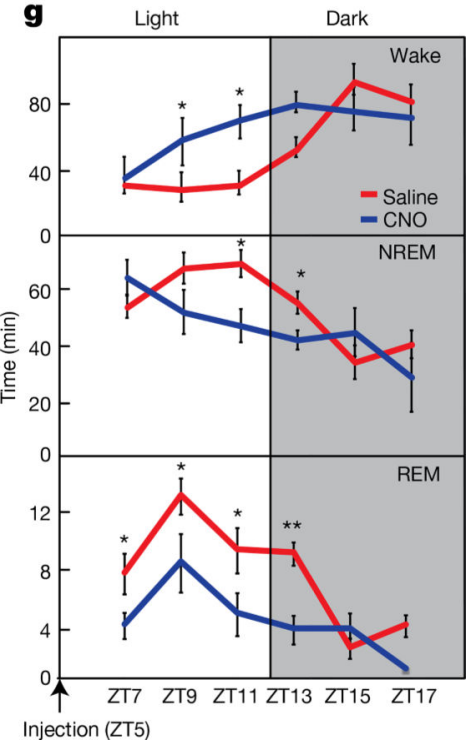

Figure 4. DREADD-dependent activation or inhibition of $\operatorname{Lhx}^{+}$neurons in the zona incerta regulates NREM and REM sleep

a, Schematic showing sleep recording conditions with saline- or CNO-injection in hM3Dqinfected (left, b-d) or hM4Di-infected (right, e-g) mice. b, hM3Dq-mCherry viral construct and injection site in Lhx6-cre mice. c, Percentage of time spent during the $12 \mathrm{~h}$ after injection in wake (top), NREM sleep (middle) and REM sleep (bottom) in saline- or CNOinjection in hM3Dq groups. d, Percentage of time spent in wake (top), NREM sleep (middle) and REM sleep (bottom) in 2-h bins following injection of saline or CNO at ZT5 in hM3Dq mice. e, hM4Di-mCherry viral construct and injection site in Lhx6-cre mice. f, 
Percentage of time spent during the $12 \mathrm{~h}$ after injection in wake (top), NREM sleep (middle) and REM sleep (bottom) in saline- or CNO-injection in hM4Di mice. g, Percentage of time in wake (top), NREM sleep (middle) and REM sleep (bottom) in 2-h bins following injection of saline or CNO at ZT5 in hM4Di mice. $* P<0.05, * * P<0.01, * * * P<0.001$; two-way ANOVA followed by Sidak's post hoc test. $n=8$ mice for all experiments. Data are mean \pm s.e.m. 